\title{
A Synthetic approach to PW2-like compounds
}

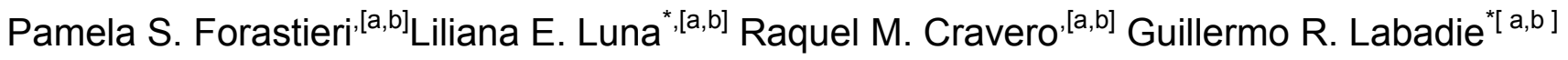

This is the peer reviewed version of the following article: P. S. Forastieri, L. E. Luna, R. M. Cravero, G. R. Labadie, A Synthetic Approach to PW2 - Like Compounds, ChemistrySelect $2020,5,1776$, which has been published in final form at https://doi.org/10.1002/slct.201903654 . This article may be used for non-commercial purposes in accordance with Wiley Terms and Conditions for Use of Self-Archived Versions. 


\section{A Synthetic approach to PW2-like compounds}

Pamela S. Forastieri, ${ }^{[a, b]}$ Liliana E. Luna*, ${ }^{[a, b]}$ Raquel M. Cravero, ${ }^{[a, b]}$ Guillermo R. Labadie*[a,b]

\footnotetext{
a Departamento de Química Orgánica, Facultad de Ciencias Bioquímicas y Farmaceúticas, Universidad Nacional del Rosario,Argentina.

b Instituto de Química Rosario (CONICET-UNR), Rosario,Argentina.

E-mail: *labadie@iquir-conicet.gov.ar

*lilianael@gmail.com

Supporting information for this article is given via a link at the end of the document.
} 
Abstract: The $9 \mathrm{H}$-xanthene derivatives, like PW2, displayed a wide spectrum of bioactivities. Herein, we reported a rapid and simple synthetic route for compounds containing the xanthenic moiety in their structure and amides. The efficient preparation of novel 1,8dioxo-2,3,4,5,6,7,8,9-octahydro-1-xanthen-9-yl- acetic acid alkyl esters by multicomponent tandem Michael-cyclization reactions starting from cyclohexanediones and alkynes. lodine and cerium (IV) ammonium nitrate were used for the oxidative aromatization step proving a series of 1,8-mono and dialkoxy-alkyl-xanthenyl-9-yl acetic acid esters in good yields. The proposed mechanism for the oxidative aromatization involves several organic transformations. The final step was the incorporation of an amide to mimic the PW2 structure that was prepared by hydrolysis of the esters, followed by the amides formation using $\mathrm{N}, \mathrm{N}$-dimethyl-1,3- propandiamine, and benzylamine.

\section{Introduction}

Molecules such as linear tricycles are ideal skeletons to produce a wide range of drugs for diverse clinical applications. Interconnected ring systems, orientation, aromaticity and heteroatoms are responsible of the ability to bind different receptors that provide a wide spectrum of bioactivities. ${ }^{[1-4]}$ Between them, xanthene derivatives present different activities including antibacterial, ${ }^{[5]}$ antiviral, ${ }^{[6]}$ antimalarial ${ }^{[7]}$ and antiinflammatory. ${ }^{[8]}$

$9 H$-xanthene derivatives, like PW2, PW3, WU5 and WU6 (Figure 1), are interesting examples of this scaffold displaying antiparasitic activity, in particular toward Plasmodium falciparum, malaria etiological agent. ${ }^{[7]}$

9,9-Dimethylxanthene derivatives recently reported by Chibale et al have shown antikinetoplastid activity against Trypanosoma cruzi, Trypanosoma brucei, and Leishmania donovani, Figure 1. ${ }^{[9]}$

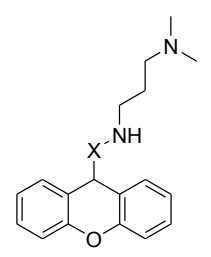<smiles>[X]c1ccc(N(CCNC)CC2c3ccccc3Oc3ccccc32)cc1</smiles><smiles>[R]c1cc([X])c2c(c1)C(C)(C)c1cc([R])cc([X])c1O2</smiles>

$\mathrm{R}=\mathrm{H}, t-\mathrm{Bu}$

$\mathrm{PW} 2, \mathrm{X}=\mathrm{C}=\mathrm{O}$
$\mathrm{PW} 3, \mathrm{X}=\mathrm{CH}_{2}$

WU5 $\mathrm{X}=\mathrm{H}$
WU6 $\mathrm{X}=\mathrm{Cl}$

9,9-Dimethylxanthenes

Figure 1. Synthetic $9 H$-xanthenes, chloroquine resistance-reversing agents.

We previously reported the synthesis of several octahydroxanthenodiones by a tandem Michael-Michael-
Cyclization reaction of 1,3-cyclohexanediones, methyl propiolate, L-proline and iodine, either in solution or in solid phase. ${ }^{[10-13]}$

Molecular iodine has played an important role in organic synthesis, ${ }^{[14]}$ being an expedient old reagent for different chemical transformations. ${ }^{[15,16]}$ Among its numerous applications, we found the systematic functionalization of Hagemann's ester derivatives that permitted the preparation of highly substituted phenols and benzenes according to Kotnis method. ${ }^{[17]}$ Likewise, the reaction of iodine in methanol was extended to prepare substituted resorcinols (olivetol) from 1,3-cyclohexanedione, and cyclohexane-1,3-diones with electron withdrawing substituents at the 2-position. The corresponding mono-methoxy resorcinol derivatives were obtained as the major products, ${ }^{[18]}$ or the substituted 2-iodomethyl- tetrahydrobenzofuran-4-ones from $\alpha$ allyl-cyclohexane-1,3-diones ones. ${ }^{[19]}$

Their synthetic utility can also be illustrated by reactions of 2 cyclohexenones with electron withdrawing group in the 4position or $\mathrm{N}$-alkyl-1,3-cyclohexadien-1-amines with iodine and sodium alkoxide undergoing regioselective iodination and aromatization. ${ }^{[20]}$

Herein, we present the synthesis of a new series of nonsymmetric hydro-xanthenodiones. The oxidative aromatization of the lateral rings of the heterocyclic core was also studied, being an efficient, user-friendly procedure to generate structurally diverse structures. ${ }^{[21,22]}$ Moreover, the collection of derivatives was extended preparing different amides, of high incidence in modern pharmaceuticals and biologically active compounds, ${ }^{[23]}$ by reaction of the xanthenic precursors with different amines.

\section{Results and Discussion}

First, we looked to expand the scope of the Michael reaction focusing on non-symmetric structures. Different 1,3cyclohexanediones were combined to introduce structural diversity in the tricycle by means of multicomponent reactions (MCR) in solution. The reaction of 1,3-cyclohexanediones 1-4, methyl propiolate and L-proline in DMSO at room temperature and subsequent $\mathrm{I}_{2} / \mathrm{EtOH}$ addition afforded the symmetric and the non-symmetric esters $\mathbf{5 - 1 5}$ in $69-77 \%$ overall yields. (Scheme 1) The 1,3-cyclohexanedione $\mathbf{4}$, that has a non-symmetrical substitution, leads to more products than the rest of the diones.

Then, the oxidative aromatization of the lateral rings of the heterocyclic core of $\mathbf{5 - 1 5}$ using molecular iodine in an alcohol solution was evaluated. 


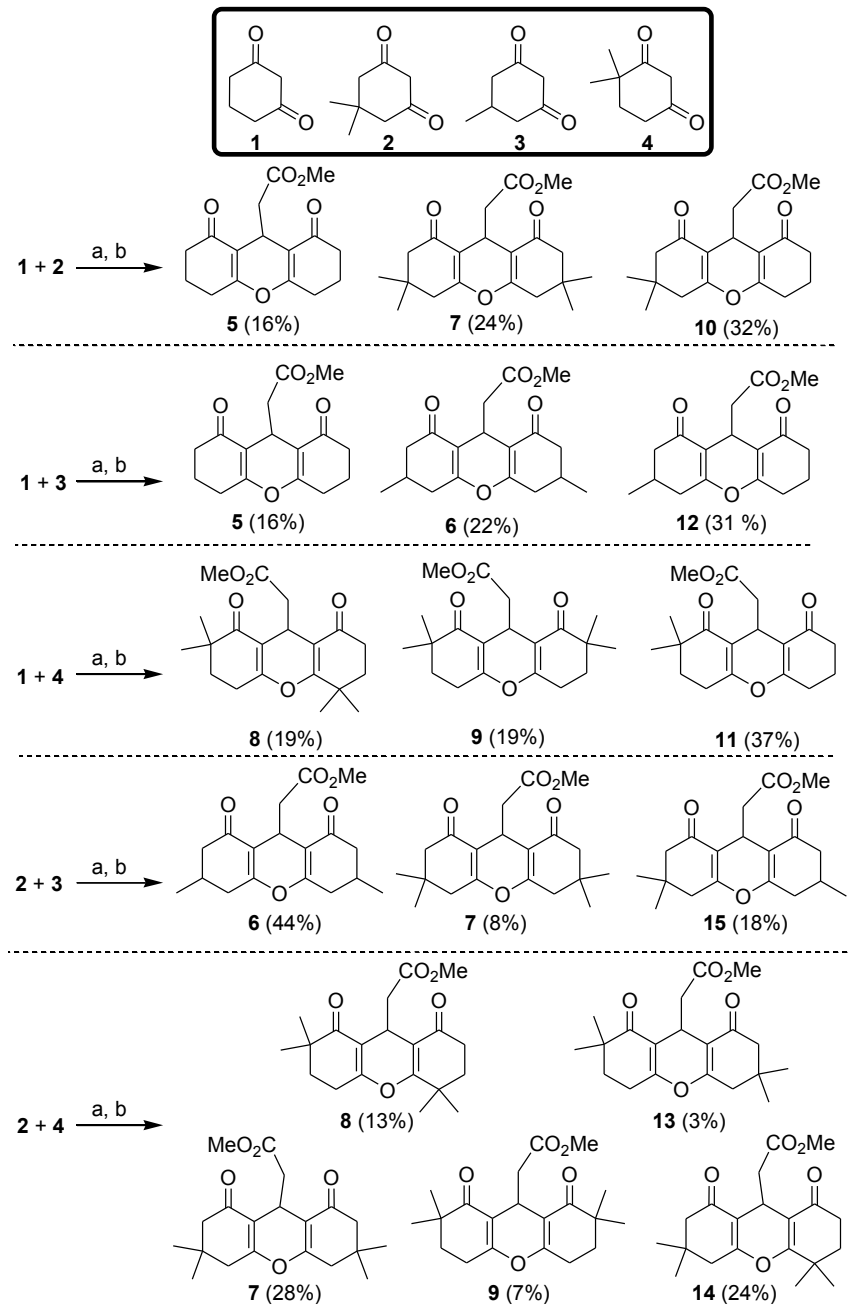

Scheme 1. Three-component synthesis of xanthenedione esters 5-15. a) Methyl propiolate, DMSO, RT, 13 days; b) $\mathrm{I}_{2}, \mathrm{MeOH}$ or $\mathrm{EtOH}, 5 \mathrm{~h}$.

The cyclohexenone portions of ester $\mathbf{5}$ undergone aromatization with iodine in refluxing methanol, being the di-oxidized ester $\mathbf{1 6}$ the major product, along with small amount of $\mathbf{2 4}$ in $75 \%$ overall yield. (Scheme 2) Alternatively, a one-pot reaction of $\mathbf{5}$ with iodine in methanol under microwave heating for 15-30 minutes provided a mixture of $\mathbf{2 4 : 1 6}$ in a 1:2 ratio with high yields (80$90 \%$ ). The reaction of the mono-substituted cyclic ketone 6 in methanol, which was less reactive than $\mathbf{5}$, provides the dimethoxy methyl ester $\mathbf{1 7}$ as only isolated product, but in low yields.

The oxidative aromatization in ethanol also produce the concomitant transesterification of the methyl acetate side chain. The addition of a catalyst such as the cerium (IV) ammonium nitrate (CAN), ${ }^{[2,25]}$ to the iodine increased the overall yield of ethyl esters 18 and 25. (Scheme 2).
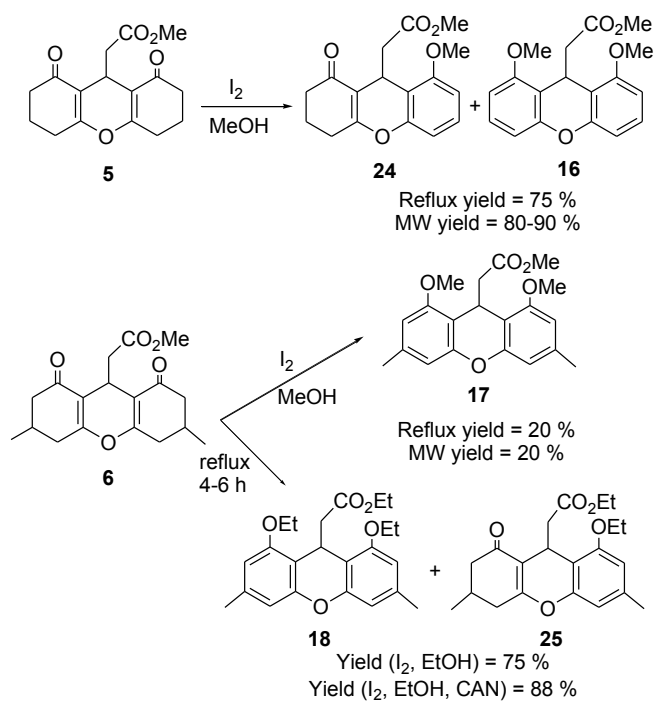

Scheme 2. Oxidative reactions on $\mathbf{5}$ and $\mathbf{6}$ with iodine.

In order to carried out the oxidation of the gem-dimethyl-ester 7, the reaction with iodine in methanol or ethanol needed CAN as catalysis, due to the lower reactivity of this substrate. Consequently, mixtures of methoxy-methyl-esters $19(20 \%)$ and $26(64 \%)$ and the corresponding ethoxy-ethyl-esters $20(25 \%)$ and $27(52 \%)$ were successfully separated during the purification by column chromatoghraphy. (Scheme 3 )
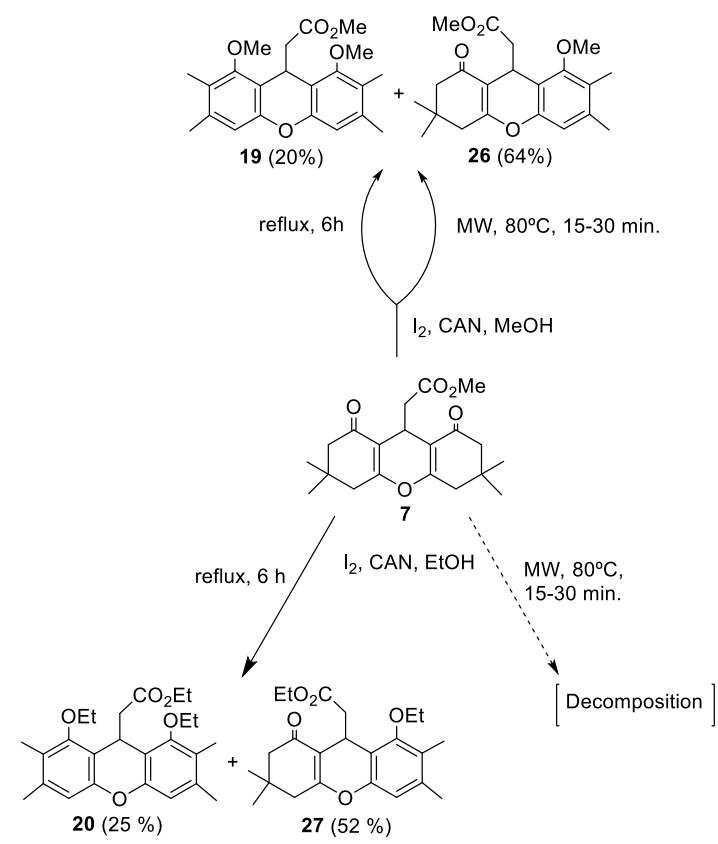

Scheme 3. Reactions of 7 with iodine, $\mathrm{CAN}, \mathrm{MeOH}$ or EtOH.

A possible mechanism for the oxidation of compound $\mathbf{7}$ is shown on Scheme 4. The addition of iodine, gives a product of $\alpha$-halogenation which undergoes a 1,2- migration of a methyl group with addition of the catalyst generating a double cross-conjugation in both cyclohexanone portions by the loss of hydrogen iodide. 
Water is then removed after addition of methanol (or ethanol) to the carbonyl group, and thus emerging the aromatic products. ${ }^{[24]}$

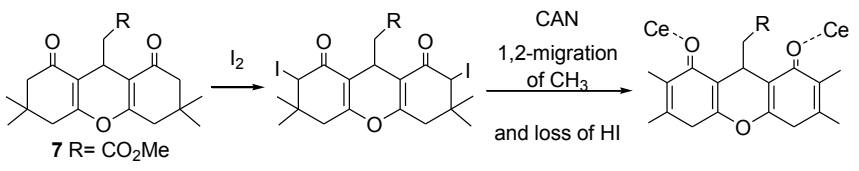<smiles>[R]CC1c2c(cc(C)c(C)c2O)Oc2cc(C)c(C)c(O)c2C1C(=O)O</smiles>

Scheme 4. Reaction mechanism of the compound 7 with iodine-cerium (IV) ammonium nitrate in methanol.

When same oxidative aromatization condition were applied on gem-dimethyl-ester $\mathbf{8}$ and $\mathbf{9}$, the reaction do not provide the expected products. That was not unexpected based on the proposed reaction mechanism, where the $\alpha$-halogenation and water loss are not possible. (Scheme 5)

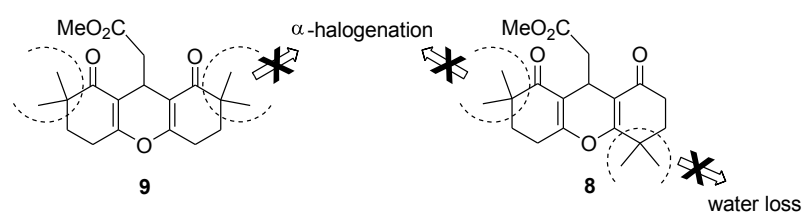

Scheme 5. Impeded reaction positions for a-halogenation and water loss.

As is shown in Scheme 6, the oxidation of the non-symmetric compounds 11-15 has also been studied. These reactions gave different mixtures of products depending on the particular ring substitution and if the aromatization occurs in one ring or both.
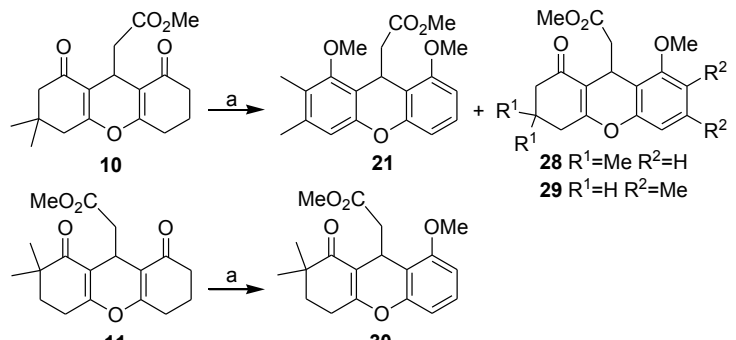<smiles>CCCC1C2=C(CCCC2=O)OC2=C1C(=O)CC(C)C2</smiles><smiles>COc1cccc2c1C(CC(C)=O)c1c(OC)cc(C)cc1O2</smiles>

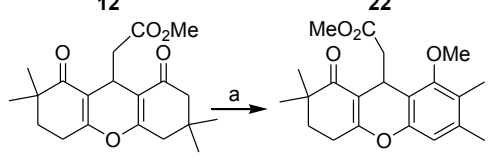

13<smiles>CCCC1C2=C(CC(C)CC2=O)OC2=C1C(=O)CC(C)(C)C2</smiles>

15<smiles>COc1cc(C)cc2c1C(CC(C)=O)c1c(OC)c(C)cc(I)c1O2</smiles>

23

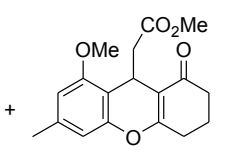

31

Scheme 6. Oxidation reactions of non symmetric compounds 10-15 with iodine-cerium (IV) ammonium nitrate in methanol. a) $\mathrm{l}_{2}, \mathrm{CAN}, \mathrm{MeOH}$; b) $\mathrm{I}_{2}$, $\mathrm{MeOH}$

Reactions carried out on compounds 11 and 13 give only products of mono-oxidation on the annular portion because one of the a-position to the carbonyl groups is blocked. Unfortunately, after different attempts, the isolation of the oxidation product of compound 14, that was expected to produce a mono-oxidized derivative, was unsuccessful.

As was mentioned before, we aim to prepare xanthenic amides derivaties that mimic PW2. Based on the previous experience of our group, the esters were transformed in carboxylic acids followed by amides formation using $\mathrm{N}$-(3(dimethylamino)propyl)amine and benzylamine. Esters 5-8 showed a low reactivity with standard reagents like aqueous $\mathrm{LiOH}, \mathrm{NaOH}$ or $\mathrm{KOH}$. To surpass that problem, the acids 35-38 were demethylated with Lil in EtOAc under reflux. ${ }^{[26]}$ Thus, 35-38 were transformed into the $\mathrm{N}$-benzyl amides $41-44$ and $\mathrm{N}$-(3(dimethylamino)propyl) amides $\mathbf{4 5 - 4 8}$ by reaction with the amines with the corresponding acids, $\mathrm{HOBt}$, and carbodiimide in dichlorometane. The mixture was maintained at $0^{\circ} \mathrm{C}$ during 1.5 hours and them warm up to room temperature. (Scheme 7) and the purification by column chromatography the amides were obtained with $53-95 \%$ yield.

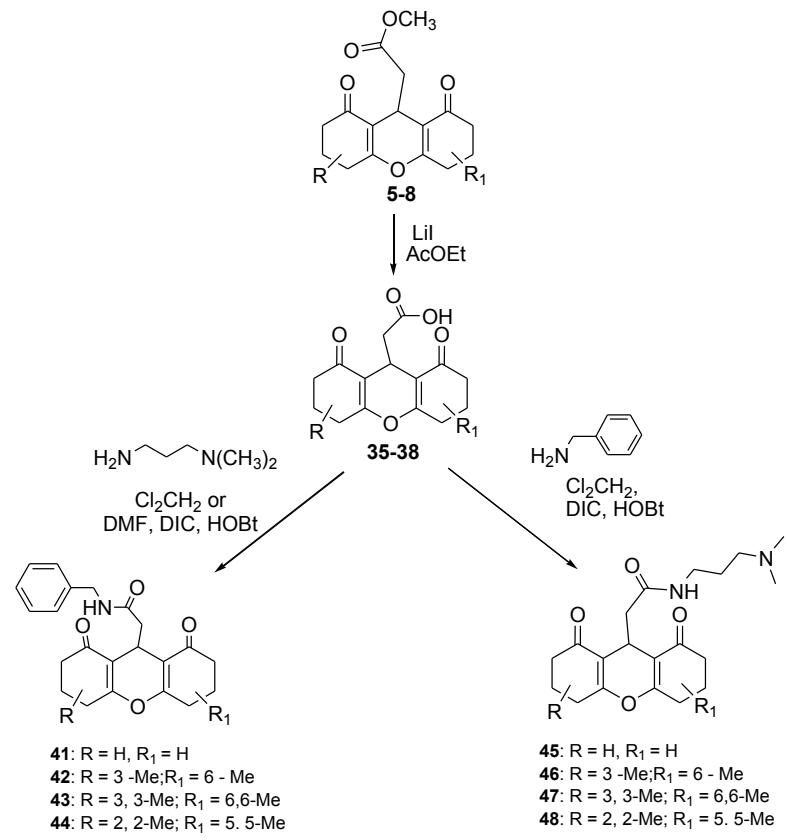

Scheme 7. Formation of amides 41-48 from the carboxylic acid 35-38

All the attempts of oxidative aromatization of amides were unsuccessful. Therefore, we decided to reach the aromatic amides $\mathbf{4 9}$ and $\mathbf{5 0}$ starting from the aromatic esters 16 and 20 through the corresponding carboxylic acids. (Scheme 8)

The acid 39 was obtained by hydrolysis using $\mathrm{LiOH}$ as a base, on the other hand, to hydrolyze the ester $\mathbf{2 0}$, the base had to be change by $\mathrm{KOH}$ since the ethyl ester did not react with $\mathrm{LiOH}$.

The amides $\mathbf{4 9}$ and $\mathbf{5 0}$ were prepared and purified in a similar way than the amides $\mathbf{4 5 - 4 8}$ with a global yield of $50-60 \%$. 


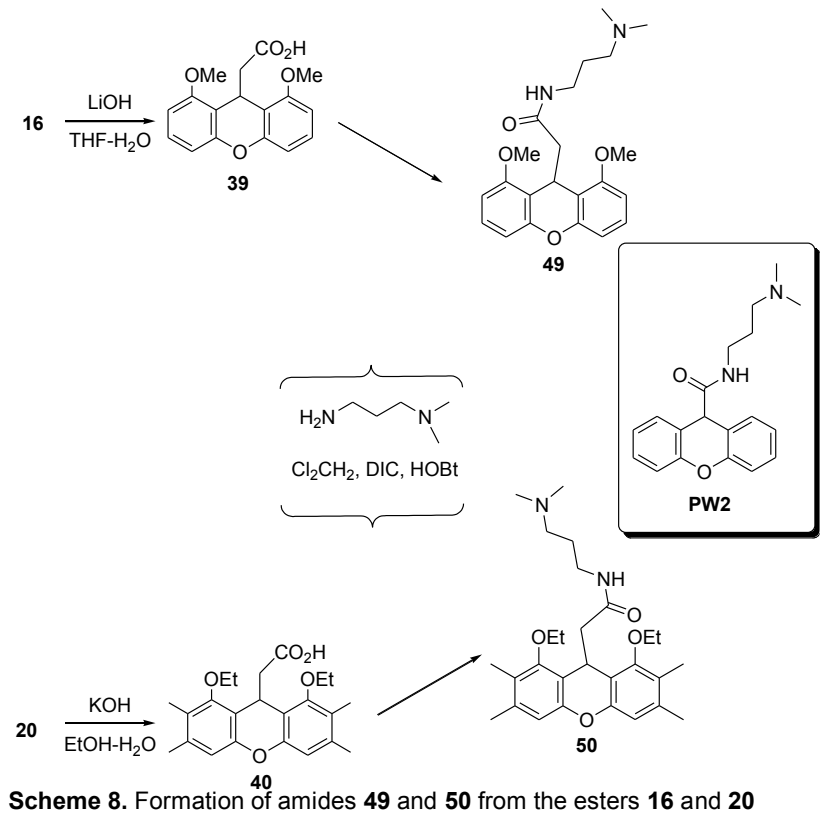

\section{Conclusions}

We have successfully developed a rapid and simple synthetic route for the efficient preparation of a series of 1,8-dioxo-2, 3, 4, $5,6,7,8,9$-octahydro-1H-xanthene -9-yl acetic acid esters 5-15 by multicomponent Michael-Michael-cyclization reactions. Molecular iodine promotes both cyclization and aromatization reactions contributing to an extension of the iodine methodology towards the formation of more complex structures.

lodine-mediated oxidative aromatization of 1,8-dioxohydroxanthene scaffold is a simple synthetic procedure for obtaining mono- and dialkoxy-xanthenyl-9-yl acetic acid esters 16-34. This oxidative aromatization involves different transformations including a-iodination, transesterification, etherification, elimination and alkyl rearrangement when it was required. Applying the reaction on non-symmetric tricycles was crucial to validate the proposed mechanism.

The final approach was to incorporate amides on the structure to mimic PW2. Compounds $\mathbf{4 9}$ and $\mathbf{5 0}$ were prepared from the corresponding acids $\mathbf{3 9}$ and $\mathbf{4 0}$ applying the same methodology used to prepared the amides 45-48.

This diversity oriented synthesis strategy will be used as the starting point of a medicinal chemistry program targeting neglected tropical diseases.

\section{Experimental Section}

General. All reactions involving air or moisture-sensitive materials, were carried out under nitrogen. Flash chromatography was performed with 300-400 mesh silica gel under slight nitrogen pressure, with increasing gradients of solvent mixtures. All chemicals and solvents are commercially available and were used after purification according to Armarego and Chai. ${ }^{[27]}$ IR spectra were recorded with a Shimadzu, Prestige 21 Model spectrophotometer, with samples as liquid films in $\mathrm{NaCl}$ for oils. NMR experiments were run in $\mathrm{CDCl}_{3}$ at $300.13 \mathrm{MHz}$ for ${ }^{1} \mathrm{H}$ NMR and at 75.4 MHz for ${ }^{13} \mathrm{C}$ NMR with a Bruker Avance-300 MHz NMR spectrometer and the corresponding solvent as internal reference standard. High-resolution mass spectrometry was carried out with a LCQTOf Bruker MicroTOF QII. Microwave heating was performed in a CEM
Discover ${ }^{\circledR}$ System using septum-sealed $10 \mathrm{~mL}$ vials for high-pressure reaction conditions with stirring and IR-monitored temperature control. Melting points (uncorrected) were measured in open capillary tubes with an Electrothermal 9100 apparatus. HPLC experiments were performed with a Hewlett Packard series 1100 system with $\mathrm{C}_{18}$ and Chiradex columns. Detection wavelength was set at $215 \mathrm{~nm}$, ref: 360 and the column was isocratically eluted with $\mathrm{MeOH}: \mathrm{H}_{2} \mathrm{O} 75: 25$ for $\mathrm{C} 18$ and 60:40 for Chiradex columns, $0.7 \mathrm{~mL} \mathrm{~min}^{-1}, c 2.0 \mathrm{mg} \mathrm{mL}^{-1}$. Optical rotations were determined using a JASCO DIP-1000 digital polarimeter in $100 \mathrm{~mm}$ cells and the sodium $\mathrm{D}$ line $(589 \mathrm{~nm})$ at temperature in solvent and concentration indicated.

Preparation of esters 5-15 (MCR): $2.2 \mathrm{mmol}$ of dione mixture and L-Pro (16 mg, $6 \mathrm{~mol} \%$ per $1.1 \mathrm{mmol}$ of dione) were dissolved in DMSO (6 mL) and methyl propiolate $(0.08 \mathrm{~mL}, 1 \mathrm{mmol})$ was added. The reaction mixture was then left for 13 days at room temperature. Finally, $\mathrm{NH}_{4} \mathrm{Cl}(1$ $\mathrm{mL})$ was added. The aqueous phase was extracted with EtOAc $(2 \times 3$ $\mathrm{mL}$ ) and the organic phase was dried with anhydrous $\mathrm{Na}_{2} \mathrm{SO}_{4}$, filtered and solvent removed under reduced pressure. For cyclization, a solution of atropisomers crude $(0.5 \mathrm{mmol})$ and iodine $(3 \mathrm{~mol} \%)$ in anhydrous $\mathrm{EtOH}(1.25 \mathrm{~mL})$ was stirred at room temperature. The reaction mixture was monitored by TLC and completed in $5 \mathrm{~h}$. The solvent was removed in vacuo, and the residue was taken up in $\mathrm{CH}_{2} \mathrm{Cl}_{2}(10 \mathrm{~mL})$ and washed with sodium thiosulphate solution $(2 \times 10 \mathrm{~mL})$, brine, and water. The combined organic layer was dried with anhydrous $\mathrm{Na}_{2} \mathrm{SO}_{4}$ and filtered, and the solvent was removed under vacuum to furnish hydroxanthenes. The residue was purified by flash chromatography using hexane/EtOAc/EtOH gradients.

Preparation of xanthenes by iodine oxidation: A solution of hydroxanthene crude $(0.5 \mathrm{mmol})$ and iodine $(635 \mathrm{mg}, 2.5 \mathrm{~mol})$ in anhydrous $\mathrm{EtOH}$ or $\mathrm{MeOH}(3 \mathrm{~mL})$ was refluxed with continuous stirring until complete conversion. The solvent was removed in vacuo, and the residue was taken up in $\mathrm{CH}_{2} \mathrm{Cl}_{2}(10 \mathrm{~mL})$ and washed with sodium thiosulphate solution $(2 \times 10 \mathrm{~mL})$, brine, and water. The organic layer was dried with anhydrous $\mathrm{Na}_{2} \mathrm{SO}_{4}$ and filtered, and the solvent was removed under vacuum to furnish xanthenes. If it is necessary to add CAN as catalyst $(0.05 \mathrm{mmol}, 10 \mathrm{~mol} \%)$.

Preparation of xanthenes by microwave-assisted iodine oxidation: A solution of hydroxanthene $(0.16 \mathrm{mmol})$ in anhydrous $\mathrm{EtOH}(3 \mathrm{~mL})$ was added into an oven-dried, $10 \mathrm{~mL}$ pressure-rated reaction vial equipped with a stirring bar. Then, iodine $(203 \mathrm{mg}, 0.80 \mathrm{mmol})$ were added. The resulting solution was stirred at $80^{\circ} \mathrm{C}$ in the microwave reactor until complete conversion. After that, the solvent was removed under reduced pressure, and the crude was dissolved in ethyl acetate $(3 \mathrm{~mL})$ and washed with sodium thiosulphate solution $(2 \times 5 \mathrm{~mL})$, brine, and water. The organic layer was dried with anhydrous $\mathrm{Na}_{2} \mathrm{SO}_{4}$ and filtered, and the solvent was removed under vacuum to furnish the product. If it is necessary to add CAN as catalyst $(0.016 \mathrm{mmol}, 10 \mathrm{~mol} \%)$.

Demethylation of esters. Preparation of carboxylic acids 35-38. Lil (1.6 mmol) was added to a solution of the ester parent $(0.16 \mathrm{mmol})$ in anhydrous EtOAc $(1.6 \mathrm{~mL})$. The mixture was refluxed with continuous stirring until complete conversion. Then it was washed with $10 \% \mathrm{Na}_{2} \mathrm{~S}_{2} \mathrm{O}_{3}$ until disappearance of the red color. The mixture was acidified to $\mathrm{pH}=4$ with $2 \mathrm{~N} \mathrm{HCl}$. The phases were separated; the aqueous phase was extracted with EtOAc $(3 \times 3 \mathrm{~mL})$. The combined organic layers were dried with anhydrous $\mathrm{Na}_{2} \mathrm{SO}_{4}$, filtered, and the solvent was removed under vacuum.

Preparation of the amides 41-44. To a solution of benzylamine $(0.142$ $\mathrm{mL}, 1.3 \mathrm{mmol})$ in anhydrous dichloromethane $(1 \mathrm{~mL})$, the acid $(1 \mathrm{mmol})$ dissolved in dichlorometane $(2.5 \mathrm{~mL})$ and HOBt $(133 \mathrm{mg}, 1 \mathrm{mmol})$ were added. The mixture was cooled to $0^{\circ} \mathrm{C}$.

$1 \mathrm{mmol}$ of diisopropylcarbodiimide (DIC) (for 41) or dicyclohexylcarbodiimide DCC (for 42-44) was then added and left with 
magnetic stirring during 90 minutes at room temperature. The formation of a solid was observed. The solvent was removed in vacuo and the crude was dissolved in THF $(2 \mathrm{~mL})$ produced the urea by-product precipitation. This precipitate led to the modification of the method which solid was filtered and washed with THF $(3 \times 1 \mathrm{~mL})$. The organic phase was concentrated under reduced pressure and the crude that was purified by column chromatography (Hexane/EtOAc, 45:55) gave the amides with good yield

Preparation of the amides 45-50. To the amine solution $(1.3 \mathrm{mmol})$ in anhydrous $\mathrm{Cl}_{2} \mathrm{CH}_{2}(3.5 \mathrm{~mL})$, acid $(1 \mathrm{mmol})$ and the $\mathrm{HOBt}(1 \mathrm{mmol})$ were added. The mixture was cooled to $0^{\circ} \mathrm{C}$ in an ice bath and was slowly added the DIC (57.1 mg, $1.2 \mathrm{mmol}$ ) and left with magnetic stirring during 90 minutes. Passed this time, the mixture was left at room temperature with stirring for 24 hours. Reaction solvent was removed under reduced pressure and the product was purified by column chromatography with solvents mixture $\left(\mathrm{Cl}_{2} \mathrm{CH}_{2} / \mathrm{MeOH} 80: 20\right)$. Yield: 77 - 94\%.

Note: The esters compounds 5-7 and 9 were obtained and described in ref. $15 b$

\section{Methyl $\quad(2,2,5,5$-tetramethyl-1,8-dioxo-2,3,4,5,6,7,8,9-octahydro-1 $H$ xanthen-9-yl)acetate (8)}

Yield 42\%. Hex:EtOAc 70 : 30. White solid. Mp: 99.2-99.7 ${ }^{\circ} \mathrm{C} . \alpha_{D}^{20.8^{\circ}}=$ +3.71 (c 0.55, $\mathrm{CHCl}_{3}$ ). HPLC: $t_{R}=5.34 \mathrm{~min}$., $\mathrm{C}_{18}\left(\mathrm{MeOH} / \mathrm{H}_{2} \mathrm{O}\right.$ 75: 25); Chiradex $\left(\mathrm{MeOH} / \mathrm{H}_{2} \mathrm{O} 60: 40\right), t_{R}=8.49 \mathrm{~min}$. and $t_{R}=12.90 \mathrm{~min}$.

IR (film): 2962, 2868 (C-H), 1732 (C=O ester), $1664(\mathrm{C}=\mathrm{O}), 1618(\mathrm{C}=\mathrm{C})$, $1381,1177,1164,1068 \mathrm{~cm}^{-1}$

${ }^{1} \mathrm{H}$ NMR $\left(300 \mathrm{MHz}, \mathrm{CDCl}_{3}, 25^{\circ} \mathrm{C}, \mathrm{TMS}\right): \delta=3.92(\mathrm{t}, J=4.4 \mathrm{~Hz}, 1 \mathrm{H}, 9-\mathrm{H})$, $3.53\left(\mathrm{~s}, 3 \mathrm{H}, \mathrm{OCH}_{3}\right), 2.60$ (dt, J = 6.8 Hz, 2H, 4-H), 2.55 (d, J = 4.4 Hz, $2 \mathrm{H}$ $\mathrm{CH}_{2} \mathrm{COOCH}_{3}$ ), 2.44 (dd, $\left.J=6.1,6.7,7.3 \mathrm{~Hz}, 2 \mathrm{H}, 7-\mathrm{H}\right), 1.93-1.79(\mathrm{~m}, 4 \mathrm{H}$, $3-\mathrm{H}, 6-\mathrm{H}), 1.28\left(\mathrm{~s}, 3 \mathrm{H}, 5-\mathrm{CH}_{3}\right), 1.22\left(\mathrm{~s}, 3 \mathrm{H}, 5-\mathrm{CH}_{3}\right), 1.13\left(\mathrm{~s}, 3 \mathrm{H}, 2-\mathrm{CH}_{3}\right)$, $1.09\left(\mathrm{~s}, 3 \mathrm{H}, 2-\mathrm{CH}_{3}\right)$.

${ }^{13} \mathrm{C}$ NMR $\left(75 \mathrm{MHz}, \mathrm{CDCl} 3,25^{\circ} \mathrm{C}, \mathrm{TMS}\right): \delta=201.7\left(\left(\mathrm{CH}_{3}\right)_{2} \mathrm{CHC}=\mathrm{O}\right)$, $196.7(\mathrm{C}=\mathrm{O}), 172.2\left(\mathrm{COOCH}_{3}\right), 170.9(4 \mathrm{~b}-\mathrm{C}), 164.0(4 \mathrm{a}-\mathrm{C}), 112.4$ and $112.0(8 \mathrm{a}-\mathrm{C}, 8 \mathrm{~b}-\mathrm{C}), 51.1\left(\mathrm{OCH}_{3}\right), 40.4(2-\mathrm{C}), 37.0\left(\mathrm{CH}_{2} \mathrm{COOCH}_{3}\right), 35.4$ (6-C), 34.4 (5-C), $34.1(7-\mathrm{C}), 33.8(3-\mathrm{C}), 26.2\left(5-\mathrm{CH}_{3}\right), 24.5\left(2-\mathrm{CH}_{3}\right), 24.4$ $\left(5-\mathrm{CH}_{3}\right), 24.1\left(2-\mathrm{CH}_{3}\right), 24.1(9-\mathrm{C}), 24.0(4-\mathrm{C})$.

HRMS (ESI): $m / z$ [M+Na] ${ }^{+}$calcd. for $\mathrm{C}_{20} \mathrm{H}_{26} \mathrm{NaO}_{5}$ : 369.16725; found: 369.16724 .

Methyl (3,3-dimethyl-1,8-dioxo-2,3,4,5,6,7,8,9-octahydro-1H-xanthen9-yl)acetate (10) was obtained as acid-derivative following the synthetic sequence of reactions on solid phase, followed by esterification as we previously reported. ${ }^{[12]}$

Methyl (2,2-dimethyl-1,8-dioxo-2,3,4,5,6,7,8,9-octahydro-1H-xanthen9-yl)acetate (11).

Yield: $37 \%$. Pale yellow oil. HPLC: $t_{R}=5.40$ min., $\mathrm{C}_{18}, \mathrm{MeOH}: \mathrm{H}_{2} \mathrm{O} 75: 25$.

IR (film): 2951, 2870 (C-H), 1732 (C=O ester), 1660 (C=O), 1622 (C=C), $1381,1175,1130,1020,952,933 \mathrm{~cm}^{-1}$.

${ }^{1} \mathrm{H}$ NMR $\left(300 \mathrm{MHz} \mathrm{CDCl}_{3}, 25^{\circ} \mathrm{C}, \mathrm{TMS}\right): \delta=3.92(\mathrm{t}, \mathrm{J}=4.3 \mathrm{~Hz}, 1 \mathrm{H}, 9-\mathrm{H})$, $3.54\left(\mathrm{~s}, 3 \mathrm{H}, \mathrm{OCH}_{3}\right), 2.58\left(\mathrm{~d}, \mathrm{~J}=4.3 \mathrm{~Hz}, 2 \mathrm{H}, \mathrm{CH}_{2} \mathrm{COOCH}_{3}\right), 2.52(\mathrm{~m}, 2 \mathrm{H}$, 4-H), 2.46-2.42 (m, 4H, 5- H, 7-H), $2.02(\mathrm{~m}, 2 \mathrm{H}, 3-\mathrm{H}), 1.85(\mathrm{~m}, 2 \mathrm{H}, 6-\mathrm{H})$, $1.12\left(\mathrm{~s}, 3 \mathrm{H}, \mathrm{CH}_{3}\right), 1.08\left(\mathrm{~s}, 3 \mathrm{H}, \mathrm{CH}_{3}\right)$.

${ }^{13} \mathrm{C}$ NMR $\left.\left(75 \mathrm{MHz}, \mathrm{CDCl}_{3}, 25^{\circ} \mathrm{C}, \mathrm{TMS}\right)\right): \delta=201.9\left(\left(\mathrm{CH}_{3}\right)_{2} \mathrm{CHC}=\mathrm{O}\right)$, $197.2(\mathrm{C}=\mathrm{O}), 172.4\left(\mathrm{COOCH}_{3}\right), 165.9(4 \mathrm{a}-\mathrm{C}), 163.9$ (4b-C), 114.1(9a-C), $112.3(8 \mathrm{a}-\mathrm{C}), 51.2\left(\mathrm{OCH}_{3}\right), 40.5(2-\mathrm{C}), 37.0(7-\mathrm{C}), 36.6\left(\mathrm{CH}_{2} \mathrm{COOCH}_{3}\right)$,
$34.8(4-\mathrm{C}), 27.2(5-\mathrm{C}), 24.2(3-\mathrm{C}), 24.1\left(\mathrm{CH}_{3}\right), 24.0\left(\mathrm{CH}_{3}\right), 23.7(9-\mathrm{C})$ $20.4(6-C)$.

HRMS (ESI): $\mathrm{m} / \mathrm{z}[\mathrm{M}+\mathrm{H}]^{+}$calcd. for $\mathrm{C}_{18} \mathrm{H}_{23} \mathrm{O}_{5}$ : 319.1545 ; found: 319.1540

Methyl (3-methyl-1,8-dioxo-2,3,4,5,6,7,8,9-octahydro-1H-xanthen-9yl)acetate (12).

Yield: $31 \%$. Pale yellow oil. $\left[\alpha_{D}^{20}\right]+0.22,\left[\alpha_{D}^{21}\right]+0.98\left(c\right.$ 1.1, $\left.\mathrm{CHCl}_{3}\right)$ HPLC: $t_{R}=4.72$ min., $\mathrm{C}_{18}, \mathrm{MeOH}: \mathrm{H}_{2} \mathrm{O} 75: 25, t_{R} 3.68,5.0 \mathrm{~min}$. Chiradex, $\left(\mathrm{MeOH} / \mathrm{H}_{2} \mathrm{O} 60: 40\right)$.

IR (film): 2953, 2875 (C-H), 1730 (C=O ester), 1660 (C=O), 1620 (C=C), $1383,1182,1132,1024,985,949,850,756 \mathrm{~cm}^{-1}$.

${ }^{1} \mathrm{H}$ NMR $\left(300 \mathrm{MHz}, \mathrm{CDCl}_{3}, 25^{\circ} \mathrm{C}, \mathrm{TMS}\right): \delta=3.92(\mathrm{t}, J=4.4 \mathrm{~Hz}, 1 \mathrm{H}, 9-\mathrm{H})$, 3.54 (s, $\left.3 \mathrm{H}, \mathrm{OCH}_{3}\right), 2.61$ (d, J = 4.3 Hz, 2H, $\left.\mathrm{CH}_{2} \mathrm{COOCH}_{3}\right), 2.64-2.09(\mathrm{~m}$, $8 \mathrm{H}, 2-\mathrm{H}, 4-\mathrm{H}, 5-\mathrm{H}, 7-\mathrm{H}$ ), $2.01(\mathrm{~m}, 3 \mathrm{H}, 3-\mathrm{H}, 6-\mathrm{H}), 1.09$ (d, J = 2.7 Hz, $3 \mathrm{H}$, $\mathrm{CH}_{3}$ ).

${ }^{13} \mathrm{C}$ NMR $\left(75 \mathrm{MHz}, \mathrm{CDCl}_{3}, 25^{\circ} \mathrm{C}, \mathrm{TMS}\right): \delta=197.1$ and $197.0(\mathrm{C}=\mathrm{O})$ $172.3\left(\mathrm{COOCH}_{3}\right), 166.0(4 \mathrm{a}-\mathrm{C}), 165.6(4 \mathrm{~b}-\mathrm{C}), 114.3(9 \mathrm{a}-\mathrm{C}), 113.8(8 \mathrm{a}-\mathrm{C})$, $51.2\left(\mathrm{OCH}_{3}\right), 45.3(4-\mathrm{C}), 45.0(7-\mathrm{C}), 37.1\left(\mathrm{CH}_{2} \mathrm{COOCH}_{3}\right), 37.0(2-\mathrm{C})$, 35.3 (5-C), 28.1 (3-C), $23.7(9-\mathrm{C}), 20.8\left(\mathrm{CH}_{3}\right), 20.4(6-\mathrm{C})$.

HRMS (ESI): $\mathrm{m} / \mathrm{z}[\mathrm{M}+\mathrm{Na}]^{+}$calcd. for $\mathrm{C}_{17} \mathrm{H}_{20} \mathrm{NaO}_{5}$ : 327.12084; found 327.12029.

Methyl (2,2,6,6-tetramethyl-1,8-dioxo-2,3,4,5,6,7,8,9-octahydro-1 Hxanthen-9-yl)acetate (13).

Yield: $3 \%$. Yellow oil. HPLC: $t_{R}=5.34 \mathrm{~min}$., $\mathrm{C}_{18},\left(\mathrm{MeOH} / \mathrm{H}_{2} \mathrm{O} 75: 25\right), t_{R}$ $=8.50,12.93$ min., Chiradex, $\left(\mathrm{MeOH} / \mathrm{H}_{2} \mathrm{O} 60: 40\right)$.

IR (film): 2960, 2860 (C-H), 1730 (ester), 1662 (C=C-C=O), 1620 (C=C), $1450,1380,1200,1175,1160,930,770 \mathrm{~cm}^{-1}$.

${ }^{1} \mathrm{H}$ NMR (300 MHz, $\left.\mathrm{CDCl}_{3}, 25^{\circ} \mathrm{C}, \mathrm{TMS}\right): \delta=3.88$ (bs, $\left.1 \mathrm{H}, 9-\mathrm{H}\right), 3.52$ (s, $\left.3 \mathrm{H}, \mathrm{OCH}_{3}\right), 2.65$ (dd, $\left.J=4.2 \mathrm{~Hz}, 1 \mathrm{H}, \mathrm{CH}_{2} \mathrm{CO}_{2} \mathrm{CH}_{3}\right), 2.50(\mathrm{dd}, \mathrm{J}=4.1 \mathrm{~Hz}$ $\left.1 \mathrm{H}, \mathrm{CH}_{2} \mathrm{CO}_{2} \mathrm{CH}_{3}\right), 2.86(\mathrm{~s}, 2 \mathrm{H}, 7-\mathrm{H}), 2.60-2.30(\mathrm{~m}, 4 \mathrm{H}, 4-\mathrm{H}, 5-\mathrm{H}), 1.82(\mathrm{~m}$, $2 \mathrm{H}, 3-\mathrm{H}), 1.29\left(\mathrm{~s}, 3 \mathrm{H}, 2-\mathrm{CH}_{3}\right), 1.27\left(\mathrm{~s}, 3 \mathrm{H}, 2-\mathrm{CH}_{3}\right), 1.08\left(\mathrm{~s}, 3 \mathrm{H}, 6-\mathrm{CH}_{3}\right)$, $1.07\left(\mathrm{~s}, 3 \mathrm{H}, 6-\mathrm{CH}_{3}\right)$.

${ }^{13} \mathrm{C}$ NMR $\left(75 \mathrm{MHz}, \mathrm{CDCl}_{3}, 25^{\circ} \mathrm{C}, \mathrm{TMS}\right): \delta=201.9(1-\mathrm{C}=\mathrm{O}), 197.1$ (8$\mathrm{C}=\mathrm{O}$ ), 172.4 (COOMe), 164.7 (4a-C), 164.5 (4b-C), 112.9 (9a-C), 113.0 (8a-C), $51.2\left(\mathrm{OCH}_{3}\right), 50.8(5-\mathrm{C}), 40.8(7-\mathrm{C}), 36.3\left(\mathrm{CH}_{2} \mathrm{COOMe}\right), 35.4$ (2 C), $34.0(3-\mathrm{C}), 32.0(6-\mathrm{C}), 29.5\left(6-\mathrm{CH}_{3}\right), 26.9\left(6-\mathrm{CH}_{3}\right), 24.5\left(2-\mathrm{CH}_{3}\right), 24.3$ (4-C), $24.1(9-\mathrm{C}), 23.8\left(2-\mathrm{CH}_{3}\right)$

HRMS (ESI): $\mathrm{m} / \mathrm{z}[\mathrm{M}+\mathrm{Na}]^{+}$calcd. for $\mathrm{C}_{20} \mathrm{H}_{26} \mathrm{NaO}_{5}$ : 369.16725; found 369.16623 .

Methyl (3,3,5,5-tetramethyl-1,8-dioxo-2,3,4,5,6,7,8,9-octahydro-1 Hxanthen-9-yl)acetate (14).

Yield: $24 \%$. Yellow oil. HPLC: $t_{R}=4.32$ min., $\mathrm{C}_{18},\left(\mathrm{MeOH} / \mathrm{H}_{2} \mathrm{O} 75: 25\right), t_{R}$ 4.81, 6.86 min., Chiradex, $\left(\mathrm{MeOH} / \mathrm{H}_{2} \mathrm{O} 60: 40\right)$.

IR (film): 2959, $2860(\mathrm{C}-\mathrm{H}), 1728$ (C=O ester), 1662 ( $\mathrm{C}=\mathrm{C}-\mathrm{C}=\mathrm{O}), 1615$ $(C=C), 1450,1380,1175,1161,1070,925,770 \mathrm{~cm}^{-1}$

${ }^{1} \mathrm{H}$ NMR $\left(300 \mathrm{MHz}, \mathrm{CDCl}_{3}, 25^{\circ} \mathrm{C}, \mathrm{TMS}\right): \delta=3.94(\mathrm{t}, \mathrm{J}=4.2 \mathrm{~Hz}, 1 \mathrm{H}, 9-\mathrm{H})$, $3.51\left(\mathrm{~s}, 3 \mathrm{H}, \mathrm{OCH}_{3}\right), 2.60\left(\mathrm{dd}, J=4.1 \mathrm{~Hz}, 2 \mathrm{H}, \mathrm{CH}_{2} \mathrm{CO}_{2} \mathrm{CH}_{3}\right), 2.44$ (t, $J=$ $6.2 \mathrm{~Hz}, 2 \mathrm{H}, 7-\mathrm{H}), 2.35$ (s, 2H, 4-H), $2.25(\mathrm{~s}, 2 \mathrm{H}, 2-\mathrm{H}), 1.85(\mathrm{~m}, 2 \mathrm{H}, 6-\mathrm{H})$, 
$1.23\left(\mathrm{~s}, 3 \mathrm{H}, 5-\mathrm{CH}_{3}\right), 1.20\left(\mathrm{~s}, 3 \mathrm{H}, 5-\mathrm{CH}_{3}\right), 1.10\left(\mathrm{~s}, 3 \mathrm{H}, 3-\mathrm{CH}_{3}\right), 1.09(\mathrm{~s}, 3 \mathrm{H}$ $\left.3-\mathrm{CH}_{3}\right)$.

${ }^{13} \mathrm{C}$ NMR $\left(75 \mathrm{MHz}, \mathrm{CDCl}_{3}, 25^{\circ} \mathrm{C}, \mathrm{TMS}\right): \delta=197.0(1-\mathrm{C}=\mathrm{O}), 196.8$ (8$\mathrm{C}=\mathrm{O}$ ), 171.0 (COOMe), 164.7 (4b-C), 164.0 (4a-C), 112.6 (9a-C), 112.2 (8a-C), $51.3\left(\mathrm{OCH}_{3}\right), 50.7$ (4-C), 40.7 (2-C), $37.0\left(\mathrm{CH}_{2} \mathrm{COOMe}\right), 35.3$ (6C), $34.5(5-\mathrm{C}), 33.8(7-\mathrm{C}), 32.0(3-\mathrm{C}), 29.5\left(3-\mathrm{CH}_{3}\right), 26.8\left(3-\mathrm{CH}_{3}\right), 26.2(5-$ $\left.\mathrm{CH}_{3}\right), 24.6\left(5-\mathrm{CH}_{3}\right), 23.8\left(\mathrm{CHCH}_{2} \mathrm{COOMe}\right)$.

HRMS (ESI): $\mathrm{m} / \mathrm{z}[\mathrm{M}+\mathrm{Na}]^{+}$calcd. for $\mathrm{C}_{20} \mathrm{H}_{26} \mathrm{NaO}_{5}$ : 369.16725; found: 369.16727.

\section{Methyl (3,3,6-trimethyl-1,8-dioxo-2,3,4,5,6,7,8,9-octahydro-1 $\mathrm{H}$ xanthen-9-yl)acetate (15)}

Yield: 9\%. Yellow oil. HPLC: $t_{R}=4.53$ min., $\mathrm{C}_{18},\left(\mathrm{MeOH} / \mathrm{H}_{2} \mathrm{O}\right.$ 75: 25$)$, Chiradex, $t_{R}$ 4.65, 7.30 min., $\left(\mathrm{MeOH} / \mathrm{H}_{2} \mathrm{O}\right.$ 60: 40).

IR (film): 2954, $2874(\mathrm{C}-\mathrm{H}), 1732$ [C(O)-OMe], 1666 (C=C-C=O), 1622 $(\mathrm{C}=\mathrm{C}), 1381,1192,1134,1028,1005,825 \mathrm{~cm}^{-1}$.

${ }^{1} \mathrm{H}$ NMR $\left(300 \mathrm{MHz}, \mathrm{CDCl}_{3}, 25^{\circ} \mathrm{C}, \mathrm{TMS}\right): \delta=3.87$ (bs, $\left.1 \mathrm{H}, 9-\mathrm{H}\right), 3.51$ (s, $\left.3 \mathrm{H}, \mathrm{OCH}_{3}\right), 2.61$ (dd, $\left.J=2.3,4.1 \mathrm{~Hz}, 2 \mathrm{H}, \mathrm{CH}_{2} \mathrm{CO}_{2} \mathrm{CH}_{3}\right), 2.58(\mathrm{~m}, 2 \mathrm{H}, 7-$ $\mathrm{H}), 2.49(\mathrm{~d}, \mathrm{~J}=3.8 \mathrm{~Hz}, 2 \mathrm{H}, 5-\mathrm{H}), 2.34(\mathrm{~s}, 2 \mathrm{H}, 4-\mathrm{H}), 2.19(\mathrm{~s}, 2 \mathrm{H}, 2-\mathrm{H})$, 2.04-1.92 (m, 1H, 6-H), 1.06 (bs, 9H, 3-gem $\left.\mathrm{CH}_{3}, 6-\mathrm{CH}_{3}\right)$.

${ }^{13} \mathrm{C} \mathrm{NMR}\left(75 \mathrm{MHz}, \mathrm{CDCl}_{3}, 25^{\circ} \mathrm{C}, \mathrm{TMS}\right): \delta=197.2(1-\mathrm{C}=\mathrm{O}, 8-\mathrm{C}=\mathrm{O}), 172.4$ $\left(\mathrm{CO}_{2} \mathrm{CH}_{3}\right), 166.1$ (4b-C), $164.5(4 \mathrm{a}-\mathrm{C}), 113.8$ and $113.2(=\mathrm{CC}=\mathrm{O}), 51.2$ $\left(\mathrm{OCH}_{3}\right), 50.8$ (2-C), 45.3 (7-C), 40.9 (4-C), $36.8\left(\mathrm{CH}_{2} \mathrm{COOMe}\right), 35.4(5-\mathrm{C})$ $32.0(\mathrm{C}-\mathrm{gemCH}), 29.4\left(3-\mathrm{CH}_{3}\right), 26.9\left(3-\mathrm{CH}_{3}\right), 28.1(6-\mathrm{C}), 23.8(9-\mathrm{C}), 20.8$ $\left(6-\mathrm{CH}_{3}\right)$.

HRMS (ESI): $m / z[M+N a]^{+}$calcd. for $\mathrm{C}_{19} \mathrm{H}_{24} \mathrm{NaO}_{5}$ : 355.1521; found: 355.1527

\section{Methyl (1,8-Dimethoxy-9H-xanthen-9-yl)-acetate (16).}

Yield: 45\%. White solid. Mp: $111.1-111.5^{\circ} \mathrm{C}$.

IR (film): 2999 (ArCH), $2945\left(\mathrm{CH}\right.$ alkane), $2837\left(\mathrm{OCH}_{3}\right), 1736(\mathrm{C}=\mathrm{O}$ ester), $1620(\mathrm{C}=\mathrm{C}), 1269(\mathrm{C}-\mathrm{O}), 1238\left(\mathrm{CH}_{3}\right) \mathrm{cm}^{-1}$.

${ }^{1} \mathrm{H}$ NMR $\left(300 \mathrm{MHz}, \mathrm{CDCl}_{3}, 25^{\circ} \mathrm{C}, \mathrm{TMS}\right): \delta=7.17(\mathrm{t}, J=8.1 \mathrm{~Hz}, 2 \mathrm{H}, 3$ $\mathrm{ArH}, 6-\mathrm{ArH}), 6.71(\mathrm{~d}, J=8.1 \mathrm{~Hz}, 2 \mathrm{H}, 4-\mathrm{ArH}, 5-\mathrm{ArH}), 6.58(\mathrm{~d}, J=8.1 \mathrm{~Hz}$, $2 \mathrm{H}, 2-\mathrm{ArH}, 7-\mathrm{ArH}), 4.80(\mathrm{t}, \mathrm{J}=5.2 \mathrm{~Hz}, 1 \mathrm{H}, 9-\mathrm{H}), 3.87\left(\mathrm{~s}, 6 \mathrm{H}, 1-\mathrm{ArOCH}_{3}, 8-\right.$ $\left.\mathrm{ArOCH}_{3}\right), 3.47\left(\mathrm{~s}, 3 \mathrm{H}, \mathrm{OCH}_{3}\right), 2.79\left(\mathrm{~d}, \mathrm{~J}=5.2 \mathrm{~Hz}, 2 \mathrm{H}, \mathrm{CH}_{2} \mathrm{CO}_{2} \mathrm{CH}_{3}\right)$.

${ }^{13} \mathrm{C} \mathrm{NMR}\left(75 \mathrm{MHz}, \mathrm{CDCl}_{3}, 25^{\circ} \mathrm{C}, \mathrm{TMS}\right): \delta=172.2\left(\mathrm{CO}_{2} \mathrm{CH}_{3}\right), 157.1$ (1-C, 8-C), 153.2 (4a-C, 4b-C), 127.9 (3-C, 6-C), 112.5 (8a-C, 9a-C), 109.0 (4C, 5-C), 104.5 (2-C, 7-C), $55.6\left(\mathrm{ArOCH}_{3}\right), \quad 51.2\left(\mathrm{OCH}_{3}\right), \quad 40.0$ $\left(\mathrm{CH}_{2} \mathrm{CO}_{2} \mathrm{Me}\right), 26.0(9-\mathrm{C})$.

HRMS (ESI): $\mathrm{m} / \mathrm{z}[\mathrm{M}+\mathrm{Na}]^{+}$calcd. for $\mathrm{C}_{18} \mathrm{H}_{18} \mathrm{NaO}_{5}: 337.10464$; found: 337.10461 .

\section{Methyl (1,8-Dimethoxy-3,6-dimethyl-9H-xanthen-9-yl)-acetate (17).}

Yield: $20 \%$. Hex:EtOAc 88 : 12. Yellow oil.

IR (film): $2949(\mathrm{CH}), 1732$ (C=O ester), 1631, $1616(\mathrm{C}=\mathrm{C}), 1574,1462$, 1219, 1153, 1099 (C-O), $822 \mathrm{~cm}^{-1}$.

${ }^{1} \mathrm{H}$ NMR $\left(300 \mathrm{MHz}, \mathrm{CDCl}_{3}, 25^{\circ} \mathrm{C}, \mathrm{TMS}\right): \delta=6.53(\mathrm{~s}, 2 \mathrm{H}, 4-\mathrm{H}, 5-\mathrm{H}), 6.39$ (s, 2H, 2-H, 7-H), 4.69 (t, J = 5.1 Hz, 1H, 9-H), $3.84\left(\mathrm{~s}, 6 \mathrm{H}, \mathrm{ArOCH}_{3}\right)$, 3.48 (s, $3 \mathrm{H}, \mathrm{OCH}_{3}$ ester), 2.74 (d, J = $\left.5.2 \mathrm{~Hz}, 2 \mathrm{H}, \mathrm{CH}_{2} \mathrm{CO}_{2} \mathrm{CH}_{3}\right), 2.32$ (s, $\left.6 \mathrm{H}, 3-\mathrm{CH}_{3}, 6-\mathrm{CH}_{3}\right)$.
${ }^{13} \mathrm{C}$ NMR $\left(75 \mathrm{MHz}, \mathrm{CDCl}_{3}, 25^{\circ} \mathrm{C}, \mathrm{TMS}\right): \delta=172.3(\mathrm{C}=\mathrm{O}), 156.9(1-\mathrm{C}, 8-$ C), 153.0 (4a,4b-C), 138.0 (3-C, 6-C), 109.6 (8a-C, 9a-C), 109.4 (4-C, 5 C), 105.5 (2-C, 7-C), 55.5 (ArOMe), $51.2\left(\mathrm{OCH}_{3}\right), 40.3\left(\mathrm{CH}_{2} \mathrm{CO}_{2} \mathrm{CH}_{3}\right)$, $25.8(9-\mathrm{C}), 21.7\left(3-\mathrm{CH}_{3}, 6-\mathrm{CH}_{3}\right)$

HRMS (ESI): $m / z \quad[M+N a]^{+}$calcd. for $\mathrm{C}_{20} \mathrm{H}_{22} \mathrm{NaO}_{5}: 365.13594$; found: 365.13621 .

\section{Ethyl (1,8-Diethoxy-3,6-dimethyl-9H-xanthen-9-yl)-acetate (18).}

Yield: 23\%. Hex:EtOAc 94 : 6. Colourless oil.

IR (film): $2926(\mathrm{CH}), 1732(\mathrm{C}=\mathrm{O}), 1605(\mathrm{C}=\mathrm{C}), 1576,1157,1114,1096$, $1035,812 \mathrm{~cm}^{-1}$.

${ }^{1} \mathrm{H}$ NMR $\left(300 \mathrm{MHz}, \mathrm{CDCl}_{3}, 25^{\circ} \mathrm{C}, \mathrm{TMS}\right): \delta=6.48$ (s, 2H, 4-Ar-H, 5-Ar-H), $6.37(\mathrm{~s}, 2 \mathrm{H}, 2-\mathrm{H}, 7-\mathrm{H}), 4.66(\mathrm{t}, J=4.6 \mathrm{~Hz}, 1 \mathrm{H}, 9-\mathrm{H}), 4.06(\mathrm{~m}, 2 \mathrm{H}$, $\mathrm{OCH}_{2} \mathrm{CH}_{3}$ ), 3.89 (q, 2H, OCH $\mathrm{CH}_{3}$ ester), 2.83 (d, $\mathrm{J}=4.6 \mathrm{~Hz}, 2 \mathrm{H}$, $\left.\mathrm{CH}_{2} \mathrm{CO}_{2} \mathrm{CH}_{2} \mathrm{CH}_{3}\right), 2.29(\mathrm{~s}, 6 \mathrm{H}, 3-\mathrm{H}, 6-\mathrm{H}), 1.45(\mathrm{t}, J=6.9 \mathrm{~Hz}, 6 \mathrm{H}$, $\mathrm{OCH}_{2} \mathrm{CH}_{3}$ ester), 1.01 (m, 3H, $\mathrm{OCH}_{2} \mathrm{CH}_{3}$ ).

${ }^{13} \mathrm{C}$ NMR $\left(\mathrm{CDCl}_{3}\right): \delta=172.0$ ( $\mathrm{C}=\mathrm{O}$ ester), 156.3 (1-C, 8-C), 152.9 (4a-C 4b-C), 137.7 (3-C, 6-C), 109.5 (8a-C, 9a-C), 109.1 (4-C, 5-C), 106.3 (2-C, 7-C), $63.6\left(\mathrm{OCH}_{2} \mathrm{CH}_{3}\right.$ ester), $59.8\left(\mathrm{OCH}_{2} \mathrm{CH}_{3}\right), 39.7\left(\mathrm{CH}_{2} \mathrm{CO}_{2} \mathrm{CH}_{3}\right), 25.8$ (9-C), $21.7\left(\mathrm{Ar}-\mathrm{CH}_{3}\right), 14.7\left(\mathrm{OCH}_{2} \mathrm{CH}_{3}\right.$ ester $), 14.3\left(\mathrm{OCH}_{2} \mathrm{CH}_{3}\right)$.

HRMS (ESI): $\mathrm{m} / \mathrm{z}[\mathrm{M}+\mathrm{Na}]^{+}$calcd. for $\mathrm{C}_{23} \mathrm{H}_{28} \mathrm{NaO}_{5}$ : 407.18290; found 407.18277 .

Note: Because of the presence of stereomers in compound 18, a greater number of peaks in the ${ }^{13} \mathrm{C}$ NMR spectra it was observed. However, the data as presented are still useful for structure determination.

Methyl (1,8-Dimethoxy-2,3,6,7-tetramethyl-9H-xanthen-9-yl)-acetate (19).

Yield: 20\%. Hex: EtOAc 92: 8. White solid, Mp: $117.4-118.1{ }^{\circ} \mathrm{C}$

IR (film): $2916(\mathrm{CH}), 1732$ (C=O ester), 1632 (C=C), 1578, 1501, 1464 $1408,1325,1275,1200,1115$ (C-O), $908,733 \mathrm{~cm}^{-1}$.

${ }^{1} \mathrm{H}$ NMR $\left(300 \mathrm{MHz}, \mathrm{CDCl}_{3}, 25^{\circ} \mathrm{C}, \mathrm{TMS}\right): \delta=6.42(\mathrm{~s}, 2 \mathrm{H}, 4-\mathrm{H}, 5-\mathrm{H}), 4.81$ (t, $J=5.8 \mathrm{~Hz}, 1 \mathrm{H}, 9-\mathrm{H}), 3.82\left(\mathrm{~s}, 6 \mathrm{H}, \mathrm{ArOCH}_{3}\right), 3.51$ (s, 3H, OCH $\mathrm{OCH}_{3}$ ester), $2.63\left(\mathrm{~d},, \mathrm{~J}=5.8 \mathrm{~Hz}, 2 \mathrm{H} \mathrm{CH} \mathrm{CO}_{2} \mathrm{CH}_{3}\right), 2.28\left(\mathrm{~s}, 6 \mathrm{H}, 3-\mathrm{CH}_{3}, 6-\mathrm{CH}_{3}\right), 2.26$ (s, $\left.6 \mathrm{H}, 2-\mathrm{CH}_{3}, 7-\mathrm{CH}_{3}\right)$

${ }^{13} \mathrm{C}$ NMR $\left(75 \mathrm{MHz}, \mathrm{CDCl}_{3}, 25^{\circ} \mathrm{C}, \mathrm{TMS}\right): \delta=172.4(\mathrm{C}=\mathrm{O}), 154.2(4 \mathrm{a}-\mathrm{C}$ 4b-C), 151.3 (1-C, 8-C), 135.9 (2-C, 7-C), 116.2 (3-C, 6-C), 110.2 (8a-C 9a-C), 106.0 (4-C, 5-C), 55.4 (ArOMe), $51.2\left(\mathrm{OCH}_{3}\right), 41.2\left(\mathrm{CH}_{2} \mathrm{CO}_{2} \mathrm{CH}_{3}\right)$, $26.4(9-\mathrm{C}), 20.3\left(3,6-\mathrm{CH}_{3}\right), 11.3\left(2,7-\mathrm{CH}_{3}\right)$.

HRMS (ESI): $\mathrm{m} / \mathrm{z}\left[\mathrm{M}+\mathrm{Na}^{+}\right.$calcd. for $\mathrm{C}_{22} \mathrm{H}_{26} \mathrm{NaO}_{5}$ : 393.16725; found 393.16696

Ethyl (1,8-Diethoxy-2,3,6,7-tetramethyl-9H-xanthen-9-yl)-acetate (20).

Yield: $25 \%$. White solid. Mp: $134.5-134.9{ }^{\circ} \mathrm{C}$

IR (film): $2924(\mathrm{CH}), 1710$ (C=O), $1634(\mathrm{C}=\mathrm{C}), 1574,1501,1412,1325$ (CH methyl), 1273, 1202, 1123, 1034, $821 \mathrm{~cm}^{-1}$.

${ }^{1} \mathrm{H}$ NMR $\left(300 \mathrm{MHz}, \mathrm{CDCl}_{3}, 25^{\circ} \mathrm{C}, \mathrm{TMS}\right): \delta=6.40(\mathrm{~s}, 2 \mathrm{H}, 4-\mathrm{H}, 5-\mathrm{H}), 4.77$ (t, $J=5.1 \mathrm{~Hz}, 1 \mathrm{H}, 9-\mathrm{H}), 4.05\left(\mathrm{~m}, 4 \mathrm{H}, 1-\mathrm{ArOCH}_{2} \mathrm{CH}_{3}, 8-\mathrm{ArOCH}_{2} \mathrm{CH}_{3}\right), 3.86$ (q, $J=7.0 \mathrm{~Hz}, 2 \mathrm{H}, \mathrm{OCH}_{2} \mathrm{CH}_{3}$, ester), $2.76(\mathrm{~d}, J=5.1 \mathrm{~Hz}, 2 \mathrm{H}$, $\mathrm{CH}_{2} \mathrm{CO}_{2} \mathrm{CH}_{2} \mathrm{CH}_{3}$ ), 2.26, 2.24 (2s, $6 \mathrm{H}$ and $\left.6 \mathrm{H}, 2,3,6,7-\mathrm{ArCH}_{3}\right), 1.44$ (t, J $\left.=6.9 \mathrm{~Hz}, 6 \mathrm{H}, 1,8-\mathrm{ArOCH}_{2} \mathrm{CH}_{3}\right), 1.02\left(\mathrm{t}, \mathrm{J}=7.1 \mathrm{~Hz}, 3 \mathrm{H}, \mathrm{OCH}_{2} \mathrm{CH}_{3}\right)$. 
${ }^{13} \mathrm{C}$ NMR $\left(75 \mathrm{MHz}, \mathrm{CDCl}_{3}, 25^{\circ} \mathrm{C}, \mathrm{TMS}\right): \delta=172.3$ ( $\mathrm{C}=\mathrm{O}$ ester), 153.6 (4a-C, 4b-C), 151.2 (1-C, 8-C), 135.6 (2-C, 7-C), 115.7 (3-C, 6-C), 110.1 (8a-C, 9a-C), 106.9 (4-C, 5-C), $63.6\left(\mathrm{ArOCH}_{2} \mathrm{CH}_{3}\right), 59.8\left(\mathrm{CO}_{2} \mathrm{CH}_{2} \mathrm{CH}_{3}\right)$, $40.6\left(\mathrm{CH}_{2} \mathrm{CO}_{2} \mathrm{Et}\right), 26.4(9-\mathrm{C}), 20.3\left(3,6-\mathrm{CH}_{3}\right), 14.9\left(\mathrm{ArOCH}_{2} \mathrm{CH}_{3}\right), 13.7$ $\left(\mathrm{CO}_{2} \mathrm{CH}_{2} \mathrm{CH}_{3}\right), 11.3\left(2,7-\mathrm{CH}_{3}\right)$.

HRMS (ESI): $m / z[M+N a]^{+}$calcd. for $\mathrm{C}_{25} \mathrm{H}_{32} \mathrm{NaO}_{5}$ : 435.21420; found: 435.21399 .

\section{Methyl (1,8-Dimethoxy-2,3-dimethyl-9H-xanthen-9-yl)-acetate (21).}

Yield: $15 \%$. Colourless oil.

IR (film): 2951 (CH Ar), $2922(\mathrm{CH}), 2850$ ( $\mathrm{CH}$ aliphatic), 1736 (C=O ester), $1625(\mathrm{C}=\mathrm{C}), 1575,1097 \mathrm{~cm}^{-1}$.

${ }^{1} \mathrm{H}$ NMR $\left(300 \mathrm{MHz}, \mathrm{CDCl} 3,25^{\circ} \mathrm{C}, \mathrm{TMS}\right): \delta=7.15(\mathrm{t}, J=8.2 \mathrm{~Hz}, 1 \mathrm{H}, 6-\mathrm{H})$, $6.75(\mathrm{~d}, J=8.1 \mathrm{~Hz}, 1 \mathrm{H}, 5-\mathrm{H}), 6.57(\mathrm{~d}, J=8.2 \mathrm{~Hz}, 1 \mathrm{H}, 7-\mathrm{H}), 6.42(\mathrm{~s}, 1 \mathrm{H}$, $4-\mathrm{H}), 4.80(\mathrm{t}, J=5.5 \mathrm{~Hz}, 1 \mathrm{H}, 9-\mathrm{H}), 3.86\left(\mathrm{~s}, 3 \mathrm{H}, \mathrm{OCH}_{3}\right), 3.83(\mathrm{~s}, 3 \mathrm{H}$, $\mathrm{OCH}_{3}$ ), 3.49 (s, $3 \mathrm{H}, \mathrm{OCH}_{3}$, ester), $2.71\left(\mathrm{~d}, J=5.6 \mathrm{~Hz}, 2 \mathrm{H}, \mathrm{CH}_{2} \mathrm{CO}_{2} \mathrm{CH}_{3}\right.$ ), $2.27\left(\mathrm{~s}, 3 \mathrm{H}, 3-\mathrm{CH}_{3}\right), 2.22\left(\mathrm{~s}, 3 \mathrm{H}, 2-\mathrm{CH}_{3}\right)$.

$\left.{ }^{13} \mathrm{C} \mathrm{NMR} \mathrm{(75} \mathrm{MHz}, \mathrm{CDCl} 3,25^{\circ} \mathrm{C}, \mathrm{TMS}\right): \delta=172.2$ (C=O ester), 157.1 (8C), 154.2 (4a-C), 153.6 (4b-C), 150.8 (1-C), 136.2 (2-C), 127.7 (6-Ar), 116.0 (3-C), 112.8 (8a-C), 109.2 (5-Ar), 104.3 (7-C), 112.8 (9a-Ar), 55.6 $\left(\mathrm{OCH}_{3}\right), 55.4\left(\mathrm{OCH}_{3}\right), 51.2\left(\mathrm{COOCH}_{3}\right), 40.6\left(\mathrm{CH}_{2} \mathrm{CO}_{2} \mathrm{CH}_{3}\right), 26.2(9-\mathrm{C})$, $20.4\left(3-\mathrm{CH}_{3}\right), 11.2\left(2-\mathrm{CH}_{3}\right)$.

HRMS (ESI): $m / z$ [M+ $\mathrm{H}^{+}$calcd. for $\mathrm{C}_{20} \mathrm{H}_{23} \mathrm{O}_{5}:$ 343.15400; found: 343.15335 .

\section{Methyl (1,8-Dimethoxy-3-methyl-9H-xanthen-9-yl)-acetate (22):}

Yield: $11 \%$. Yellow oil.

IR (film): 2953 (CH Ar), $2918(\mathrm{CH}), 2849$ ( $\mathrm{CH}$ aliphatic), 1734 (C=O ester), $1605(\mathrm{C}=\mathrm{C}), 1586,1263,1085 \mathrm{~cm}^{-1}$.

$\left.{ }^{1} \mathrm{H} \mathrm{NMR} \mathrm{(300} \mathrm{MHz,} \mathrm{CDCl}_{3}, 25^{\circ} \mathrm{C}, \mathrm{TMS}\right): \delta=7.15(\mathrm{t}, J=8.3 \mathrm{~Hz}, 1 \mathrm{H}, 6-\mathrm{H})$, $6.70(\mathrm{~d}, J=8.3 \mathrm{~Hz}, 1 \mathrm{H}, 5-\mathrm{H}), 6.58(\mathrm{~d}, J=8.3 \mathrm{~Hz}, 1 \mathrm{H}, 7-\mathrm{H}), 6.54(\mathrm{~s}, 1 \mathrm{H}, 4-$ $\mathrm{H}), 6.40(\mathrm{~s}, 1 \mathrm{H}, 2-\mathrm{H}), 4.74(\mathrm{t}, J=5.1 \mathrm{~Hz}, 1 \mathrm{H}, 9-\mathrm{H}), 3.86\left(\mathrm{~s}, 3 \mathrm{H}, \mathrm{OCH}_{3}\right)$, $3.85\left(\mathrm{~s}, 3 \mathrm{H}, \mathrm{OCH}_{3}\right), 3.47\left(\mathrm{~s}, 3 \mathrm{H}, \mathrm{OCH}_{3}\right.$, ester), $2.76(\mathrm{~d}, J=5.2 \mathrm{~Hz}, 2 \mathrm{H}$, $\left.\mathrm{CH}_{2} \mathrm{CO}_{2} \mathrm{CH}_{3}\right), 2.32\left(\mathrm{~s}, 3 \mathrm{H}, 3-\mathrm{CH}_{3}\right)$.

${ }^{13} \mathrm{C}$ NMR $\left(75 \mathrm{MHz}, \mathrm{CDCl}_{3}, 25^{\circ} \mathrm{C}, \mathrm{TMS}\right): \delta=172.2$ (C=O ester), 157.1 (1C), 156.8 (8-C), 153.3 (4b-C), 152.8 (4a-C), 138.1 (3-C), 127.7 (6-C), 116.0 (3-C), 112.8 (8a-C), 109.2 (5-Ar), 104.3 (7-C), 112.8 (9a-Ar), 55.6 $\left(\mathrm{OCH}_{3}\right), 55.4\left(\mathrm{OCH}_{3}\right), 51.2\left(\mathrm{COOCH}_{3}\right), 40.6\left(\mathrm{CH}_{2} \mathrm{CO}_{2} \mathrm{CH}_{3}\right), 25.9(9-\mathrm{C})$, $21.7\left(3-\mathrm{CH}_{3}\right)$.

HRMS (ESI): $m / z$ [M+ Na] ${ }^{+}$calcd. for $\mathrm{C}_{19} \mathrm{H}_{20} \mathrm{NaO}_{5}$ : 351.1207; found: 351.1208

Methyl (1,8-Dimethoxy-2,3,6-trimethyl-9H-xanthen-9-yl)-acetate (23):

Yield: $15 \%$. Pale yellow oil.

IR (film): 2957 (CH Ar), 2929 (CH), 2849 (CH aliphatic), 1734 (C=O ester), $1630(\mathrm{C}=\mathrm{C}), 1387\left(\mathrm{CH}\right.$ methyl), $1111 \mathrm{~cm}^{-1}$.

${ }^{1} \mathrm{H}$ NMR $\left(300 \mathrm{MHz}, \mathrm{CDCl}_{3}, 25^{\circ} \mathrm{C}, \mathrm{TMS}\right): \delta=6.59(\mathrm{~s}, 1 \mathrm{H}, 5-\mathrm{H}), 6.41(\mathrm{~s}$ $1 \mathrm{H}, 4-\mathrm{H}), 6.31(\mathrm{~s}, 1 \mathrm{H}, 7-\mathrm{H}), 4.75(\mathrm{t}, J=5.5 \mathrm{~Hz}, 1 \mathrm{H}, 9-\mathrm{H}), 3.84(\mathrm{~s}, 3 \mathrm{H}$, $\left.\mathrm{OCH}_{3}\right), 3.82\left(\mathrm{~s}, 3 \mathrm{H}, \mathrm{OCH}_{3}\right), 3.48\left(\mathrm{~s}, 3 \mathrm{H}, \mathrm{OCH}_{3}\right.$, ester), $2.68(\mathrm{~d}, J=5.6 \mathrm{~Hz}$, $\left.2 \mathrm{H}, \mathrm{CH}_{2} \mathrm{CO}_{2} \mathrm{CH}_{3}\right), 2.32\left(\mathrm{~s}, 3 \mathrm{H}, 6-\mathrm{CH}_{3}\right), 2.26\left(\mathrm{~s}, 3 \mathrm{H}, 3-\mathrm{CH}_{3}\right), 2.20(\mathrm{~s}, 3 \mathrm{H}$, $\left.2-\mathrm{CH}_{3}\right)$. $\left.{ }^{13} \mathrm{C} \mathrm{NMR} \mathrm{(75} \mathrm{MHz}, \mathrm{CDCl}_{3}, 25^{\circ} \mathrm{C}, \mathrm{TMS}\right): \delta=172.3$ ( $\mathrm{C}=\mathrm{O}$ ester), 156.8, 154.2 (1-C) (8-C), 153.3, 151.0 (4a-C), (4b-C), 137.0 (6-Ar), 136.0 (2-Ar), 116.0 (3-Ar), 119.9 (8a-C), 109.5 (5-Ar), 105.5 (7-Ar), 110.5 (9a-Ar), 55.5 $\left(\mathrm{OCH}_{3}\right), 55.4\left(\mathrm{OCH}_{3}\right), 51.2\left(\mathrm{COOCH}_{3}\right), 40.7\left(\mathrm{CH}_{2} \mathrm{CO}_{2} \mathrm{CH}_{3}\right), 26.1(9-\mathrm{C})$, $21.7\left(6-\mathrm{CH}_{3}\right), 20.4\left(3-\mathrm{CH}_{3}\right), 11.2\left(2-\mathrm{CH}_{3}\right)$

HRMS (ESI): $\mathrm{m} / \mathrm{z} \quad[\mathrm{M}+\mathrm{Na}]^{+}$calcd. for $\mathrm{C}_{21} \mathrm{H}_{24} \mathrm{NaO}_{5}: 379.1516$; found: 379.1512 .

\section{Methyl (8-methoxy-1-oxo-2,3,4,9-tetrahydro-1H-xanthen-9-yl) acetate} (24).

\section{Yield: $30 \%$. Yellow oil.}

IR (film): 2949, $2839(\mathrm{CH}), 1736$ (C=O, ester), $1645(\mathrm{C}=\mathrm{O}), 1616(\mathrm{C}=\mathrm{C})$, 1587, 1387 (CH methyl), 1267, 1240, 1184, 1080 (C-O), 1003, $783 \mathrm{~cm}^{-1}$.

${ }^{1} \mathrm{H}$ NMR $\left(300 \mathrm{MHz}, \mathrm{CDCl}_{3}, 25^{\circ} \mathrm{C}\right.$, TMS): $\delta=7.15$ (t, $J=8.3 \mathrm{~Hz}, 1 \mathrm{H}, 6-\mathrm{Ar}-$ H), $6.65(\mathrm{~d}, J=8.1 \mathrm{~Hz}, 1 \mathrm{H}, 5-\mathrm{Ar}-\mathrm{H}), 6.63(\mathrm{~d}, J=8.1 \mathrm{~Hz}, 1 \mathrm{H}, 7-\mathrm{Ar}-\mathrm{H}), 4.37$ (t, $J=4.6 \mathrm{~Hz}, 1 \mathrm{H}, 9-\mathrm{H}), 3.84\left(\mathrm{~s}, 3 \mathrm{H}, \mathrm{Ar}-\mathrm{OCH}_{3}\right), 3.49\left(\mathrm{~s}, 3 \mathrm{H}, \mathrm{OCH}_{3}\right), 2.73$ (d, $\left.J=4.8 \mathrm{~Hz}, 2 \mathrm{H}, \mathrm{CH}_{2} \mathrm{CO}_{2} \mathrm{CH}_{3}\right), 2.63-2.31(\mathrm{~m}, 4 \mathrm{H}, 2-\mathrm{H}, 4-\mathrm{H}), 2.05(\mathrm{~m}$, $2 \mathrm{H}, 3-\mathrm{H})$.

${ }^{13} \mathrm{C} \mathrm{NMR}\left(75 \mathrm{MHz}, \mathrm{CDCl}_{3}, 25^{\circ} \mathrm{C}, \mathrm{TMS}\right): \delta=197.4(\mathrm{C}=\mathrm{O}), 172.2(\mathrm{C}(\mathrm{O})$ ester), 168.2 (4a-C), 157.2 (8-Ar), 151.4 (4b-Ar), 128.0 (6-Ar), 112.7, 112.6 (8a-Ar, 9a-C), 108.7 (5-C), 106.2 (7-C), $55.6\left(\mathrm{ArOCH}_{3}\right), 51.2$ $\left(\mathrm{OCH}_{3}\right), 38.4\left(\mathrm{CH}_{2} \mathrm{CO}_{2} \mathrm{CH}_{3}\right), 37.0(2-\mathrm{C}), 27.8(4-\mathrm{C}), 24.7$ (9-C), 20.6 (3-C).

HRMS (ESI): $m / z[M+N a]^{+}$calcd. for $\mathrm{C}_{17} \mathrm{H}_{18} \mathrm{NaO}_{5}:$ 325.10464; found: 325.10410

Ethyl (8-ethoxy-3,6-dimethyl-1-oxo-2,3,4,9-tetrahydro-1H-xanthen-9yl)acetate (25).

Yield: 65\%. Hex: EtOAc 95: 5. Yellow oil.

IR (film): 2980, 2931 (CH), 1734, 1732 (C=O), 1672 (C=C), 1574, 1371 (CH of methyl), 1288, 1178, 112, 1031, $814 \mathrm{~cm}^{-1}$.

${ }^{1} \mathrm{H}$ NMR $\left(300 \mathrm{MHz}, \mathrm{CDCl}_{3}, 25^{\circ} \mathrm{C}, \mathrm{TMS}\right): \delta=6.88(\mathrm{~s}, 1 \mathrm{H}, 7-\mathrm{Ar}-\mathrm{H}), 6.42(\mathrm{~s}$ $1 \mathrm{H}, \quad 5-\mathrm{Ar}-\mathrm{H}), \quad 4.34 \quad\left(\mathrm{~s}, \quad 2 \mathrm{H}, \quad \mathrm{CH}_{2} \mathrm{CO}_{2} \mathrm{CH}_{2} \mathrm{CH}_{3}\right), \quad 4.20-3.94 \quad(\mathrm{~m}, \quad 4 \mathrm{H}$, $\mathrm{CH}_{2} \mathrm{CO}_{2} \mathrm{CH}_{2} \mathrm{CH}_{3}, \mathrm{OCH}_{2} \mathrm{CH}_{3}$ ), 3.01 (dd, $J=16.5,6.4 \mathrm{~Hz}, 1 \mathrm{H}, 2-\mathrm{H}$ ), 2.96 $(\mathrm{dd}, J=16.5,7.2 \mathrm{~Hz}, 1 \mathrm{H}, 2-\mathrm{H}), 2.67(\mathrm{t}, J=5.2 \mathrm{~Hz}, 1 \mathrm{H}$, $\left.\mathrm{CHCH}_{2} \mathrm{CO}_{2} \mathrm{CH}_{2} \mathrm{CH}_{3}\right), 2.51-2.38(\mathrm{~m}, 1 \mathrm{H}, 4-\mathrm{H}), 2.40\left(\mathrm{~s}, 3 \mathrm{H}, 6-\mathrm{CH}_{3}\right), 2.35-$ $2.20(\mathrm{~m}, 2 \mathrm{H}, 3-\mathrm{H}, 4-\mathrm{H}), 1.40\left(\mathrm{t}, 3 \mathrm{H}, \mathrm{J}=7.0 \mathrm{~Hz}, \mathrm{OCH}_{2} \mathrm{CH}_{3}\right) ; 1,24(\mathrm{t}, 3 \mathrm{H}, J$ $\left.=7.1 \mathrm{~Hz}, \mathrm{CO}_{2} \mathrm{CH}_{2} \mathrm{CH}_{3}\right), 1.06\left(\mathrm{~d}, J=6.7 \mathrm{~Hz}, 3 \mathrm{H}, 3-\mathrm{CH}_{3}\right)$.

$\left.{ }^{13} \mathrm{C} \mathrm{NMR} \mathrm{(75} \mathrm{MHz,} \mathrm{CDCl}_{3}, 25^{\circ} \mathrm{C}, \mathrm{TMS}\right): \delta=192.4$ (C=O, ketone), 172.4 (C=O, ester), 155.5 (8-C), 155.1 (4b-C), 147.4 (4a-C), 140.4 (6-C), 121.3 (9a-C), 116.4 (8a-C), 105.9 (5-C), 104.8 (7-C), $63.7\left(\mathrm{OCH}_{2} \mathrm{CH}_{3}\right), 60.7$ $\left(\mathrm{C}(\mathrm{O}) \mathrm{OCH}_{2} \mathrm{CH}_{3}\right), 45.8(2-\mathrm{C}), 41.3(4-\mathrm{C}), 31.0\left(\mathrm{CH}_{2} \mathrm{C}(\mathrm{O}) \mathrm{OCH}_{2} \mathrm{CH}_{3}\right), 29.7$ (9-C), $26.5(3-\mathrm{C}), 22.5\left(6-\mathrm{CH}_{3}\right), 20.1\left(3-\mathrm{CH}_{3}\right), 14.6\left(\mathrm{OCH}_{2} \mathrm{CH}_{3}\right), 14.2$ $\left(\mathrm{C}(\mathrm{O}) \mathrm{OCH}_{2} \mathrm{CH}_{3}\right)$.

HRM (ESI): $m / z$ [M+ Na] ${ }^{+}$calcd. for $\mathrm{C}_{21} \mathrm{H}_{26} \mathrm{NaO}_{5}: 381.16725$; found 381.16667 .

Methyl (8-methoxy-3,3,6,7-tetramethyl-1-oxo-2,3,4,9-tetrahydro-1 $\mathrm{H}$ xanthen-9-yl)acetate (26).

Yield: 64\%. Hex:EtOAc 86: 14. Yellow oil.

IR (film): $2951(\mathrm{CH}), 1738$ (C=O ester), 1651(C=O ketone), 1582, 1499, 1466, 1385, 1286, 1194, 1105 (C-O), $822 \mathrm{~cm}^{-1}$. 
${ }^{1} \mathrm{H}$ NMR $\left(300 \mathrm{MHz}, \mathrm{CDCl}_{3}, 25^{\circ} \mathrm{C}, \mathrm{TMS}\right): \delta=6.46(\mathrm{~s}, 1 \mathrm{H}, 5-\mathrm{H}), 4.33(\mathrm{t}, \mathrm{J}$ $=4.8 \mathrm{~Hz}, 1 \mathrm{H}, 9-\mathrm{H}), 3.79\left(\mathrm{~s}, 3 \mathrm{H}, \mathrm{ArOCH}_{3}\right), 3.47\left(\mathrm{~s}, 3 \mathrm{H}, \mathrm{OCH}_{3}\right.$ ester $), 2.72$ $\left(\mathrm{m}, 2 \mathrm{H}, \mathrm{CH}_{2} \mathrm{CO}_{2} \mathrm{CH}_{3}\right), 2.48(\mathrm{~d}, \mathrm{~J}=7.4 \mathrm{~Hz}, 2 \mathrm{H}, 4-\mathrm{H}), 2.30(\mathrm{~s}, 2 \mathrm{H}, 2-\mathrm{H})$, $2.25\left(\mathrm{~s}, 3 \mathrm{H}, 6-\mathrm{Ar}-\mathrm{CH}_{3}\right), 2.13\left(\mathrm{~s}, 3 \mathrm{H}, 7-\mathrm{Ar}-\mathrm{CH}_{3}\right), 1.14\left(\mathrm{~s}, 3 \mathrm{H}, 3-\mathrm{CH}_{3}\right), 1.11$ (s, $\left.3 \mathrm{H}, 3-\mathrm{CH}_{3}\right)$.

${ }^{13} \mathrm{C}$ RMN $\left(75 \mathrm{MHz}, \mathrm{CDCl}_{3}, 25^{\circ} \mathrm{C}, \mathrm{TMS}\right): \delta=197.3$ (C=O ketone), 172.4 (C=O ester), 166.8 (4a-C), 154.4 (4b-C), 149.2 (8-C), 136.5 (7-C), 115.8 (6-C), 111.4 (9a-C), 109.8 (8a-C), 107.8 (5-C), 55.5 (ArOMe), 51.2 $\left(\mathrm{OCH}_{3}\right), 50.9(2-\mathrm{C}), 41.6(4-\mathrm{C}), 38.6\left(\mathrm{CH}_{2} \mathrm{CO}_{2} \mathrm{CH}_{3}\right), 32.0$ (3-C), 29.6, 27.0 (3-gemCH$), 24.9(9-\mathrm{C}), 20.3\left(6-\mathrm{CH}_{3}\right), 11.1\left(7-\mathrm{CH}_{3}\right)$

HRMS (ESI): $\mathrm{m} / \mathrm{z}[\mathrm{M}+\mathrm{H}]^{+}$calcd. for $\mathrm{C}_{21} \mathrm{H}_{27} \mathrm{O}_{5}:$ 359.18530; found: 359.18409 .

\section{Ethyl (8-ethoxy-3,3,6,7-tetramethyl-1-oxo-2,3,4,9-tetrahydro-1 $\mathrm{H}$ xanthen-9-yl)acetate (27).}

Yield: $52 \%$. Pale yellow oil.

IR (film): 2951 ( $\mathrm{CH} \mathrm{Ar}), 2926(\mathrm{CH}), 2849$ (CH aliphatic), $1734(\mathrm{C}=\mathrm{O}$ ester), $1653(\mathrm{C}=\mathrm{O}), 1558,1387\left(\mathrm{CH}\right.$ methyl), 1099, $1037 \mathrm{~cm}^{-1}$.

${ }^{1} \mathrm{H}$ NMR $\left(300 \mathrm{MHz}, \mathrm{CDCl}_{3}, 25^{\circ} \mathrm{C}, \mathrm{TMS}\right): \delta=6.44(\mathrm{~s}, 1 \mathrm{H}, 5-\mathrm{Ar}-\mathrm{H}), 4.32(\mathrm{t}$, $J=4.5 \mathrm{~Hz}, 1 \mathrm{H}, 9-\mathrm{H}), 4.01$ (dd, $\left.J=2.5,7.0 \mathrm{~Hz}, 2 \mathrm{H}, \mathrm{OCH}_{2} \mathrm{CH}_{3}\right), 3.89$ (dd, $J=2.7,7.0 \mathrm{~Hz}, 2 \mathrm{H}, \mathrm{OCH}_{2} \mathrm{CH}_{3}$, ester), $2.78(\mathrm{dd}, J=4.5,6.0 \mathrm{~Hz}, 2 \mathrm{H}$, $\mathrm{CH}_{2} \mathrm{CO}_{2} \mathrm{CH}_{2} \mathrm{CH}_{3}$ ), 2.48 (d, J = 4.5 Hz, 2H,4-H), 2.31 (s, 2H, 2-H), 2.23 (s, $3 \mathrm{H}, 6-\mathrm{CH}_{3}$ ), 2.11 (s, 3H, 7-CH $\mathrm{CH}_{3}, 1.41$ (t, 3H, J = 7.0 Hz, $\mathrm{ArOCH}_{2} \mathrm{CH}_{3}$ ), $1.15\left(\mathrm{~s}, 3 \mathrm{H}, 3-\mathrm{CH}_{3}\right), 1.11\left(\mathrm{~s}, 3 \mathrm{H}, 3-\mathrm{CH}_{3}\right), 1.04(\mathrm{t}, J=7.1 \mathrm{~Hz}, 3 \mathrm{H}$, $\left.\mathrm{OCH}_{2} \mathrm{CH}_{3}\right)$.

${ }^{13} \mathrm{C}$ NMR (75 MHz, $\left.\mathrm{CDCl}_{3}, 25^{\circ} \mathrm{C}, \mathrm{TMS}\right): \delta=197.4(\mathrm{C}=\mathrm{O}), 172.2(\mathrm{C}=\mathrm{O}$ ester), 166.7 (4a-C), 153.8 (4b-C), 149.2 (8-C), 136.3 (7-Ar), 115.5 (6-Ar) 111.4 (9a-C), 109.7 (8a-C), 108.5 (5-Ar), $63.7\left(\mathrm{ArOCH}_{2} \mathrm{CH}_{3}\right), 59.8$ $\left(\mathrm{COOCH}_{2} \mathrm{CH}_{3}\right), 51.0$ (2-C), 41.6 (4-C), $38.4\left(\mathrm{CH}_{2} \mathrm{CO}_{2} \mathrm{Et}\right), 32.0$ (3-C), 29.6 27.1 (3-gemCH$), 25.0$ (9-C), $20.3\left(6-\mathrm{CH}_{3}\right), 14.9\left(\mathrm{ArOCH}_{2} \mathrm{CH}_{3}\right), 13.9$ $\left(\mathrm{CH}_{2} \mathrm{CH}_{3}\right), 11.1\left(7-\mathrm{CH}_{3}\right)$

HRMS-ESI: $\mathrm{m} / \mathrm{z}$ [M+ Na] ${ }^{+}$calcd. for $\mathrm{C}_{23} \mathrm{H}_{30} \mathrm{NaO}_{5}$ : 409.19855; found: 409.19824.

\section{Methyl (8-methoxy-3,3-dimethyl-1-oxo-2,3,4,9-tetrahydro-1 H- xanthen-9-yl)acetate (28).}

Yield: $35 \%$. Colourless oil.

IR (film): 2951 ( $\mathrm{CH} \mathrm{Ar}), 2926(\mathrm{CH}), 2849$ (CH aliphatic), $1736(\mathrm{C}=\mathrm{O}$ ester), 1647(C=O), 1583, 1387 (CH methyl), 1267, 1080, $1029 \mathrm{~cm}^{-1}$.

${ }^{1} \mathrm{H} \mathrm{NMR}\left(300 \mathrm{MHz}, \mathrm{CDCl}_{3}, 25^{\circ} \mathrm{C}, \mathrm{TMS}\right): \delta=7.15(\mathrm{t}, J=8.2 \mathrm{~Hz}, 1 \mathrm{H}, 6-\mathrm{H})$, $6.64(\mathrm{~d}, J=8.2 \mathrm{~Hz}, 1 \mathrm{H}, 5-\mathrm{H}), 6.63(\mathrm{~d}, J=8.2 \mathrm{~Hz}, 1 \mathrm{H}, 7-\mathrm{H}), 4.34(\mathrm{t}, J=$ $4.4 \mathrm{~Hz}, 1 \mathrm{H}, 9-\mathrm{H}$ ), 3.84 (s, 3H, OCH 3 ), 3.46 (s, 3H, OCH 3 , ester), 2.77 (d, $\left.J=4.6 \mathrm{~Hz}, 2 \mathrm{H}, \mathrm{CH}_{2} \mathrm{CO}_{2} \mathrm{CH}_{3}\right), 2.46(\mathrm{~s}, 2 \mathrm{H}, 4-\mathrm{H}), 2.30(\mathrm{~s}, 2 \mathrm{H}, 2-\mathrm{H}), 1.13$ (s, 3H, 3- $\left.\mathrm{CH}_{3}\right), 1.11\left(\mathrm{~s}, 3 \mathrm{H}, 3-\mathrm{CH}_{3}\right)$.

${ }^{13} \mathrm{C} \mathrm{NMR}\left(75 \mathrm{MHz}, \mathrm{CDCl}_{3}, 25^{\circ} \mathrm{C}, \mathrm{TMS}\right): \delta=197.3(\mathrm{C}=\mathrm{O}), 172.3(\mathrm{C}=\mathrm{O}$ ester), 166.7 (4a-C), 157.2 (8-C), 151.5 (4b-C), 128.0 (6-Ar), 108.8 (5-Ar), 106.2 (7-Ar), 112.6 (8a-C), $111.4(9 a-A r), 55.6\left(\mathrm{OCH}_{3}\right), 51.2\left(\mathrm{COOCH}_{3}\right)$, 50.9 (2-C), 41.5 (4-C), $38.0\left(\mathrm{CH}_{2} \mathrm{CO}_{2} \mathrm{CH}_{3}\right), 32.0$ (3-C), 29.6, 26.9 (3gem $\left.\mathrm{CH}_{3}\right), 24.8(9-\mathrm{C})$.

HRMS (ESI): $\mathrm{m} / \mathrm{z}[\mathrm{M}+\mathrm{H}]^{+}$calcd. for $\mathrm{C}_{19} \mathrm{H}_{23} \mathrm{O}_{5}: 331.15400$; found 331.15413.

\footnotetext{
Methyl (8-methoxy-6,7-dimethyl-1-oxo-2,3,4,9-tetrahydro-1Hxanthen-9-yl)acetate (29).
}

Yield: $19 \%$. Colourless oil.

IR (film): 2956 ( $\mathrm{CH} \mathrm{Ar}), 2921(\mathrm{CH}), 2850$ (CH aliphatic), 1735 (C=O ester), $1650(\mathrm{C}=\mathrm{O}), 1387$ ( $\mathrm{CH}$ methyl), $1103 \mathrm{~cm}^{-1}$.

${ }^{1} \mathrm{H} \mathrm{NMR}\left(300 \mathrm{MHz}, \mathrm{CDCl}_{3}, 25^{\circ} \mathrm{C}, \mathrm{TMS}\right): \delta=6.46(\mathrm{~s}, 1 \mathrm{H}, 5-\mathrm{Ar}-\mathrm{H}), 4.36(\mathrm{t}$, $J=4.9 \mathrm{~Hz}, 1 \mathrm{H}, 9-\mathrm{H}), 3.79\left(\mathrm{~s}, 3 \mathrm{H}, \mathrm{ArOCH}_{3}\right), 3.50\left(\mathrm{~s}, 3 \mathrm{H}, \mathrm{OCH}_{3}\right), 2.69$ (dd, $\left.J=4.8 \mathrm{~Hz}, 2 \mathrm{H}, \mathrm{CH}_{2} \mathrm{CO}_{2} \mathrm{CH}_{3}\right), 2.68-2.54(\mathrm{~m}, 2 \mathrm{H}, 4-\mathrm{H}), 2.48-2.38(\mathrm{~m}, 2 \mathrm{H}$, 2-H), 2.25 (s, 3H, 7-CH3), $2.14\left(\mathrm{~s}, 3 \mathrm{H}, 6-\mathrm{CH}_{3}\right), 2.04(\mathrm{~m}, 2 \mathrm{H}, 3-\mathrm{H})$.

${ }^{13} \mathrm{C}$ NMR $\left(75 \mathrm{MHz}, \mathrm{CDCl}_{3}, 25^{\circ} \mathrm{C}, \mathrm{TMS}\right): \delta=197.5(\mathrm{C}=\mathrm{O}), 172.3(\mathrm{C}=\mathrm{O}$ ester), 168.3 (4a-C), 154.3 (4b-C), 149.1 (8-C), 136.5 (7-Ar), 115.8 (6-C) 112.7 (9a-C), 109.9 (8a-C), $107.8(5-\mathrm{C}), 55.5\left(\mathrm{ArOCH}_{3}\right), 51.2\left(\mathrm{COOCH}_{3}\right)$, $38.9\left(\mathrm{CH}_{2} \mathrm{CO}_{2} \mathrm{CH}_{3}\right), 37.1$ (2-C), 27.9 (4-C), 24.8 (9-C), 20.6 (3-C), 20.3 $\left(6-\mathrm{CH}_{3}\right), 11.1\left(7-\mathrm{CH}_{3}\right)$.

HRMS (ESI): $m / z[M+H]^{+}$calcd. for $\mathrm{C}_{19} \mathrm{H}_{23} \mathrm{O}_{5}$ : 331.15400; found 331.15452

Methyl (8-methoxy-2,2-dimethyl-1-oxo-2,3,4,9-tetrahydro-1 Hxanthen-9-yl) acetate (30).

Yield: $79 \%$. Colourless oil.

IR (film): $2955(\mathrm{CH} \mathrm{Ar}), 2920(\mathrm{CH}), 2849(\mathrm{CH}$ aliphatic), $1732(\mathrm{C}=\mathrm{O}$ ester), $1651(\mathrm{C}=\mathrm{O}), 1590,1387\left(\mathrm{CH}\right.$ methyl), 1267, $1080 \mathrm{~cm}^{-1}$.

${ }^{1} \mathrm{H} \mathrm{NMR}\left(300 \mathrm{MHz}, \mathrm{CDCl}_{3}, 25^{\circ} \mathrm{C}, \mathrm{TMS}\right): \delta=7.15(\mathrm{t}, \mathrm{J}=8.2 \mathrm{~Hz}, 1 \mathrm{H}, 6-\mathrm{H})$, 6.64 and 6.62 (t, $J=8.2 \mathrm{~Hz}, 1 \mathrm{H}, 5-\mathrm{H}), \quad(\mathrm{t}, J=8.2 \mathrm{~Hz}, 1 \mathrm{H}, 7-\mathrm{H}), 4.34$ (t, J $=4.7 \mathrm{~Hz}, 1 \mathrm{H}, 9-\mathrm{H}), 3.84\left(\mathrm{~s}, 3 \mathrm{H}, \mathrm{ArOCH}_{3}\right), 3.48\left(\mathrm{~s}, 3 \mathrm{H}, \mathrm{OCH}_{3}\right), 2.69(\mathrm{dd}, \mathrm{J}$ $\left.=4.7 \mathrm{~Hz}, J=1.1 \mathrm{~Hz}, 2 \mathrm{H}, \mathrm{CH}_{2} \mathrm{CO}_{2} \mathrm{CH}_{3}\right), 2.60(\mathrm{~m}, 2 \mathrm{H}, 4-\mathrm{H}), 1.88(\mathrm{~m}, 2 \mathrm{H}$, $3-\mathrm{H}), 1.17,1.10\left(2 \mathrm{~s}, 3 \mathrm{H}\right.$ and $\left.3 \mathrm{H}, 2-\mathrm{gemCH}_{3}\right)$.

${ }^{13} \mathrm{C}$ NMR $\left(75 \mathrm{MHz}, \mathrm{CDCl}_{3}, 25^{\circ} \mathrm{C}, \mathrm{TMS}\right): \delta=202.1(\mathrm{C}=\mathrm{O}), 172.3(\mathrm{C}=\mathrm{O}$ ester), 166.0 (4a-C), 157.2 (8-Ar), 151.4 (4b-Ar), 127.9 (6-Ar), 112.7 (8a$\mathrm{Ar}), 110.7$ (9a-C), 108.6 and 106.1(5-Ar, 7-Ar), $55.6\left(\mathrm{ArOCH}_{3}\right), 51.2$ $\left(\mathrm{COOCH}_{3}\right), 40.4(2-\mathrm{C}), 38.5\left(\mathrm{CH}_{2} \mathrm{CO}_{2} \mathrm{CH}_{3}\right), 34.2$ (3-C), 24.9, 24.2 (2gemCH $\left.\mathrm{CH}_{3}\right), 24.7$ (4-C), 25.1 (9-C).

HRMS (ESI): $m / z[M+H]^{+}$calcd. for $\mathrm{C}_{19} \mathrm{H}_{23} \mathrm{O}_{5}$ : 331.15400; found: 331.15406 .

Methyl (8-methoxy-6-methyl-1-oxo-2,3,4,9-tetrahydro-1H-xanthen-9yl)acetate (31).

Yield: $10 \%$. Colourless oil.

IR (film): 2951 ( $\mathrm{CH} \mathrm{Ar}), 2918(\mathrm{CH}), 2849$ (CH aliphatic), 1736 (C=O ester), $1645(\mathrm{C}=\mathrm{O}), 1582,1387\left(\mathrm{CH}\right.$ methyl), $1092 \mathrm{~cm}^{-1}$.

${ }^{1} \mathrm{H}$ NMR $\left(300 \mathrm{MHz}, \mathrm{CDCl}_{3}, 25^{\circ} \mathrm{C}, \mathrm{TMS}\right): \delta=6.47(\mathrm{~s}, 1 \mathrm{H}, 5-\mathrm{H}), 6.44(\mathrm{~s}$, $1 \mathrm{H}, 7-\mathrm{H}$ ), 4.32 (t, J = 4.4 Hz, 1H, 9-H), 3.82 (s, 3H, $\left.\mathrm{ArOCH}_{3}\right), 3.50$ (s, 3H, $\left.\mathrm{OCH}_{3}\right), 2.71$ (dd, $\left.J=4.6 \mathrm{~Hz}, J=1.6 \mathrm{~Hz}, 2 \mathrm{H}, \mathrm{CH}_{2} \mathrm{CO}_{2} \mathrm{CH}_{3}\right), 2.79(\mathrm{~m}, 4 \mathrm{H}$, 2- $\mathrm{H}$ and 4-H), 2.31 (s, 3H, $\left.\mathrm{ArCH}_{3}\right), 2.05(\mathrm{~m}, 2 \mathrm{H}, 3-\mathrm{H})$.

${ }^{13} \mathrm{C}$ NMR $\left(75 \mathrm{MHz}, \mathrm{CDCl}_{3}, 25^{\circ} \mathrm{C}, \mathrm{TMS}\right): \delta=197.4(\mathrm{C}=\mathrm{O}), 172.3(\mathrm{C}=\mathrm{O}$ ester), 168.3 (4a-C), 156.9 (8a-Ar), 151.1 (4b-Ar), 138.3 (6-Ar), 112.7 (9a-C), 109.1 (5-Ar), $107.3(7-\mathrm{Ar}), 55.6\left(\mathrm{ArOCH}_{3}\right), 51.2\left(\mathrm{COOCH}_{3}\right), 38.5$ (2-C), $37.0\left(\mathrm{CH}_{2} \mathrm{CO}_{2} \mathrm{CH}_{3}\right), 27.0$ (4-C), $24.6(9-\mathrm{C}), 21.6\left(\mathrm{ArCH}_{3}\right), 20.6$ (3C).

HRMS (ESI): $\mathrm{m} / \mathrm{z}[\mathrm{M}+\mathrm{H}]^{+}$calcd. for $\mathrm{C}_{18} \mathrm{H}_{21} \mathrm{O}_{5}$ : 317.3835 ; found 317.13713

Methyl (8-methoxy-2,2,6,7-tetramethyl-1-oxo-2,3,4,9-tetrahydro-1 Hxanthen-9-yl)acetate (32) 
Yield: $59 \%$. Colourless oil.

IR (film): 2947 (CH Ar), $2918(\mathrm{CH}), 2849$ (CH aliphatic), 1734 (C=O ester), 1647 ( $\mathrm{C}=\mathrm{O}), 1541,1387$ ( $\mathrm{CH}$ methyl), $1105 \mathrm{~cm}^{-1}$.

${ }^{1} \mathrm{H}$ NMR $\left(300 \mathrm{MHz}, \mathrm{CDCl}_{3}, 25^{\circ} \mathrm{C}, \mathrm{TMS}\right): \delta=6.46(\mathrm{~s}, 1 \mathrm{H}, 5-\mathrm{H}), 4.33(\mathrm{t}, J$ $=4.8 \mathrm{~Hz}, 1 \mathrm{H}, 9-\mathrm{H}), 3.80\left(\mathrm{~s}, 3 \mathrm{H}, \mathrm{ArOCH}_{3}\right), 3.49\left(\mathrm{~s}, 3 \mathrm{H}, \mathrm{OCH}_{3}\right), 2.66-2.60$ (m, $4 \mathrm{H}, 4-\mathrm{H}$ and $\left.\mathrm{CH}_{2} \mathrm{CO}_{2} \mathrm{CH}_{3}\right), 2.25\left(\mathrm{~s}, 3 \mathrm{H}, 7-\mathrm{CH}_{3}\right), 2.12\left(\mathrm{~s}, 3 \mathrm{H}, 6-\mathrm{CH}_{3}\right)$, 1.92- $1.83(\mathrm{~m}, 2 \mathrm{H}, 3-\mathrm{H}), 1.16$ and $1.10\left(2 \mathrm{~s}, 3 \mathrm{H}\right.$ and $\left.3 \mathrm{H}, 2-g e m \mathrm{CH}_{3}\right)$.

${ }^{13} \mathrm{C}$ NMR $\left(75 \mathrm{MHz}, \mathrm{CDCl}_{3}, 25^{\circ} \mathrm{C}, \mathrm{TMS}\right): \delta=202.1(\mathrm{C}=\mathrm{O}), 172.4(\mathrm{C}=\mathrm{O}$ ester), 166.2 (4a-C), 149.1 (8-Ar), 154.4 (4b-Ar), 136.6 (7-Ar), 115.7(6$\mathrm{Ar}), 110.7$ (9a-C), 109.9 (8a-Ar), 107.7 (5-Ar), $55.5\left(\mathrm{ArOCH}_{3}\right), 51.2$ $\left(\mathrm{COOCH}_{3}\right), 40.3(2-\mathrm{C}), 39.0\left(\mathrm{CH}_{2} \mathrm{CO}_{2} \mathrm{CH}_{3}\right), 34.3(3-\mathrm{C}), 24.8(4-\mathrm{C}), 24.9$ and $24.2\left(2-\right.$ gem $\left.\mathrm{CH}_{3}\right), 25.2(9-\mathrm{C}), 20.3\left(6-\mathrm{CH}_{3}\right), 11.1\left(7-\mathrm{CH}_{3}\right)$

HRMS (ESI): $m / z[M+N a]^{+}$calcd. for $\mathrm{C}_{21} \mathrm{H}_{26} \mathrm{NaO}_{5}$ : 381.1678; found: 381.1708 .

Methyl (8-methoxy-3,3,6-trimethyl-1-oxo-2,3,4,9-tetrahydro-1 Hxanthen-9-yl)acetate (33).

Yield: $25 \%$. Colourless oil.

IR (film): $2953(\mathrm{CH} \mathrm{Ar}), 2920(\mathrm{CH}), 2849$ (CH aliphatic), $1736(\mathrm{C}=\mathrm{O}$ ester), $1649(\mathrm{C}=\mathrm{O}), 1583,1385$ ( $\mathrm{CH}$ methyl), 1092, $1036 \mathrm{~cm}^{-1}$.

${ }^{1} \mathrm{H}$ NMR $\left(300 \mathrm{MHz}, \mathrm{CDCl}_{3}, 25^{\circ} \mathrm{C}, \mathrm{TMS}\right): \delta=6.47(\mathrm{~s}, 1 \mathrm{H}, 5-\mathrm{H}), 6.44(\mathrm{~s}$, $1 \mathrm{H}, 7-\mathrm{H}), 4.29(\mathrm{t}, J=4.6 \mathrm{~Hz}, 1 \mathrm{H}, 9-\mathrm{H}), 3.82\left(\mathrm{~s}, 3 \mathrm{H}, \mathrm{ArOCH}_{3}\right), 3.47$ (s, 3H, $\mathrm{OCH}_{3}$ ), 2.76 (dd, $\left.J=4.6 \mathrm{~Hz}, J=1.1 \mathrm{~Hz}, 2 \mathrm{H}, \mathrm{CH}_{2} \mathrm{CO}_{2} \mathrm{CH}_{3}\right), 2.44$ (s, 2H, 4$\mathrm{H}), 2.31\left(\mathrm{~s}, 3 \mathrm{H}, 6-\mathrm{CH}_{3}\right), 2.29(\mathrm{~s}, 2 \mathrm{H}, 2-\mathrm{H}), 1.13,1.10(2 \mathrm{~s}, 3 \mathrm{H}$ and $3 \mathrm{H} 3-$ gem $\mathrm{CH}_{3}$ ).

${ }^{13} \mathrm{C}$ NMR $\left(75 \mathrm{MHz}, \mathrm{CDCl}_{3}, 25^{\circ} \mathrm{C}, \mathrm{TMS}\right): \delta=197.3(\mathrm{C}=\mathrm{O}), 172.4(\mathrm{C}=\mathrm{O}$ ester), 166.8 (4a-C), 157.0 (8-Ar), 151.2 (4b-Ar), 138.3 (6-Ar), 111.6 (9aC), 109.4 (8a-Ar), 109.2 (5-Ar), 107.3(7-Ar), $55.6\left(\mathrm{ArOCH}_{3}\right), 51.2$ $\left(\mathrm{COOCH}_{3}\right), 50.9$ (2-C), 41.5 (4-C), $38.1\left(\mathrm{CH}_{2} \mathrm{CO}_{2} \mathrm{CH}_{3}\right), 32.0$ (3-C), 29.6, $27.0\left(3-g e m \mathrm{CH}_{3}\right), 24.6(9-\mathrm{C}), 21.6\left(6-\mathrm{CH}_{3}\right)$.

HRMS (ESI): $m / z[M+H]^{+}$calcd. for $\mathrm{C}_{20} \mathrm{H}_{25} \mathrm{O}_{5}$ : 345.16965; found: 345.16796 .

\section{Methyl (8-methoxy-3,6,7-trimethyl-1-oxo-2,3,4,9-tetrahydro-1 $\mathrm{H}$ - xanthen-9-yl)acetate (34).}

Yield: $12 \%$. Pale yellow oil.

IR (film): 2951 (CH Ar), 2921(CH), 2849 (CH aliphatic), 1734 (C=O ester), $1660(\mathrm{C}=\mathrm{O}), 1387$ ( $\mathrm{CH}$ methyl), $1092 \mathrm{~cm}^{-1}$.

${ }^{1} \mathrm{H}$ NMR $\left(300 \mathrm{MHz}, \mathrm{CDCl}_{3}, 25^{\circ} \mathrm{C}, \mathrm{TMS}\right): \delta=6.90(\mathrm{~s}, 1 \mathrm{H}, 5-\mathrm{H}), 4.31(\mathrm{t}, J$ $=4.0 \mathrm{~Hz}, 1 \mathrm{H}, 9-\mathrm{H}), 3.86\left(\mathrm{~s}, 3 \mathrm{H}, \mathrm{OCH}_{3}\right), 3.63\left(\mathrm{~s}, 3 \mathrm{H}, \mathrm{OCH}_{3}\right.$, ester), 3.02 (br.s, $2 \mathrm{H}, 4-\mathrm{H}$ ), 2.72 (dd, $J=4.0 \mathrm{~Hz}, 2 \mathrm{H}, \mathrm{CH}_{2} \mathrm{CO}_{2} \mathrm{CH}_{3}$ ), 2.50 (s, $2 \mathrm{H}, 2-\mathrm{H}$ ), 2.46 (br. s, $1 \mathrm{H}, 3-\mathrm{H}$ ), 2.25 (s, 3H, Ar- $\left.\mathrm{CH}_{3}\right), 2.13$ (s, 3H, Ar- $\left.\mathrm{CH}_{3}\right), 1.15$ (br.s, 3H, 3- $\mathrm{CH}_{3}$ ).

${ }^{13} \mathrm{C} \mathrm{NMR}\left(75 \mathrm{MHz}, \mathrm{CDCl}_{3}, 25^{\circ} \mathrm{C}, \mathrm{TMS}\right): \delta=193.5(\mathrm{C}=\mathrm{O}), 172.5(\mathrm{C}=\mathrm{O}$ ester), 171.0 (4a-C), 149.1 (8-C), 155.3 (4b-C), 136.7 (7-C), 116.4 (6-Ar) 115.8 (9a-Ar), 105.3 (8a-C), 105.1(5-Ar), $55.4\left(\mathrm{OCH}_{3}\right), 51.2\left(\mathrm{COOCH}_{3}\right)$, 48.7 (4-C), 45.4 (2-C), $38.1\left(\mathrm{CH}_{2} \mathrm{CO}_{2} \mathrm{CH}_{3}\right), 28.0\left(3-\mathrm{CH}_{3}\right), 24.9(9-\mathrm{C}), 22.6$ (3-C), $20.4\left(6-\mathrm{CH}_{3}\right), 11.0\left(7-\mathrm{CH}_{3}\right)$.

HRMS (ESI): $m / z[M+N a]^{+}$calcd. for $\mathrm{C}_{20} \mathrm{H}_{24} \mathrm{NaO}_{5}$ : 367.1516; found: 367.1519 .
Note: The acids compounds 36-38 were obtained by solid phase synthesis and described in ref. 13.

(2,2,5,5-Tetramethyl-1,8-dioxo-2,3,4,5,6,7,8,9-octahydro-1 H-xanthen9 -yl)-acetic acid (38).

Yield: 99\%. Colourless oil.

IR (film): 3200 (O-H acid), 2965, $2968(\mathrm{CH}), 1713(\mathrm{C}=\mathrm{O}), 1661$ (O=C$\mathrm{C}=\mathrm{C}), 1614(\mathrm{C}=\mathrm{C}), 1176(\mathrm{C}-\mathrm{O}) \mathrm{cm}^{-1}$.

${ }^{1} \mathrm{H} \mathrm{NMR}\left(300 \mathrm{MHz} \mathrm{CDCl}_{3}, 25^{\circ} \mathrm{C}, \mathrm{TMS}\right): \delta=3.92(\mathrm{t}, J=4.1 \mathrm{~Hz}, 1 \mathrm{H}, 9-\mathrm{H})$, 2.58-2.43 (m, 4H, 4- H, 7-H), $2.52\left(\mathrm{~d}, \mathrm{~J}=4.1 \mathrm{~Hz}, 2 \mathrm{H}, \mathrm{CH}_{2} \mathrm{COOH}\right), 1.87-$ $1.79(\mathrm{~m}, 4 \mathrm{H}, 3-\mathrm{H}, 6-\mathrm{H}), 1.24\left(\mathrm{~s}, 3 \mathrm{H}, 5-\mathrm{CH}_{3}\right), 1.23\left(\mathrm{~s}, 3 \mathrm{H}, 5-\mathrm{CH}_{3}\right), 1.11(\mathrm{~s}$, $\left.3 \mathrm{H}, 2-\mathrm{CH}_{3}\right), 1.09\left(\mathrm{~s}, 3 \mathrm{H}, 2-\mathrm{CH}_{3}\right)$.

$\left.{ }^{13} \mathrm{C} \mathrm{NMR} \mathrm{(75} \mathrm{MHz}, \mathrm{CDCl}_{3}, 25^{\circ} \mathrm{C}, \mathrm{TMS}\right): \delta=202.6(1-\mathrm{C}), 197.9$ (8-C), $177.5(\mathrm{COOH}), 171.5(4 \mathrm{~b}-\mathrm{C}), 164.5(4 \mathrm{a}-\mathrm{C}), 112.5$ (8a-C), $112.1(9 \mathrm{a}-\mathrm{C})$, $40.5(2-\mathrm{C}), 38.6\left(\mathrm{CH}_{2} \mathrm{COOH}\right), 35.2(6-\mathrm{C}), 34.6(5-\mathrm{C}), 34.0(3-\mathrm{C}), 33.8$ (7C), $26.1\left(5-\mathrm{CH}_{3}\right), 24.6\left(2-\mathrm{CH}_{3}\right), 24.4\left(5-\mathrm{CH}_{3}\right), 24.2\left(2-\mathrm{CH}_{3}\right), 24.0(4-\mathrm{C})$, $23.8(9-\mathrm{C})$.

HRMS (ESI): $\quad m / z[M+2 \mathrm{Na}]^{+}$calcd. for $\mathrm{C}_{19} \mathrm{H}_{24} \mathrm{Na}_{2} \mathrm{O}_{5}$ : 377.13354 ; found: 377.13245 .

Hydrolysis of dimethoxy aryl methyl ester. Preparation of 1,8dimethoxy-9H-xanthen-9-yl)-acetic acid (39). Aqueous $\mathrm{LiOH}$ solution $(1.0 \mathrm{M}, 4 \mathrm{~mL}, 4 \mathrm{mmol})$ was added to a solution of methyl ester $(0.21$ $\mathrm{mmol})$ in THF $(4.0 \mathrm{~mL})$. The reaction mixture was stirred at room temperature for 3 days. After removal of THF in vacuo, the residue was redissolved with $\mathrm{EtAcO}$. The aqueous layers were cautiously acidified to $\mathrm{pH}=4$ with $10 \% \mathrm{HCl}$ and extracted with EtAcO $(3 \times 5 \mathrm{~mL})$. The combined organic layers were dried over anhydrous sodium sulphate, filtered and the solvent was removed under vacuum.

Yield: $99 \%$. White solid. Mp: $188.6-189.6{ }^{\circ} \mathrm{C}$.

IR (film): 3479 (OH), 2924 (CH), 2850 ( $\mathrm{CH}$ aliphatic), 1705 (C=O ester), 1620, 1582 (C=CAr), 1269 (C-OAr), $1238\left(\mathrm{CH}_{3}-\mathrm{OAr}\right) \mathrm{cm}^{-1}$.

${ }^{1} \mathrm{H}$ NMR $\left(300 \mathrm{MHz}, \mathrm{CDCl}_{3}, 25^{\circ} \mathrm{C}, \mathrm{TMS}\right): \delta=7.17$ (t, $J=8.2 \mathrm{~Hz}, 2 \mathrm{H}, 3-\mathrm{Ar}-$ $\mathrm{H}, 6-\mathrm{Ar}-\mathrm{H}$ ), 6.70 (d, J = 8.2 Hz, 2H, 2-Ar-H, 7-Ar-H), 6.57 (d, J = 8.2 Hz, $2 \mathrm{H}, 4-\mathrm{Ar}-\mathrm{H}, 5-\mathrm{Ar}-\mathrm{H}), 4.83(\mathrm{t}, J=5.5 \mathrm{~Hz}, 1 \mathrm{H}, 9-\mathrm{H}), 3.79\left(\mathrm{~s}, 6 \mathrm{H}, \mathrm{OCH}_{3}\right)$, $2.76\left(\mathrm{~d}, \mathrm{~J}=5.5 \mathrm{~Hz}, 2 \mathrm{H}, \mathrm{CH}_{2} \mathrm{CO}_{2} \mathrm{H}\right)$.

${ }^{13} \mathrm{C}$ NMR $\left(75 \mathrm{MHz}, \mathrm{CDCl}_{3}, 25^{\circ} \mathrm{C}, \mathrm{TMS}\right): \delta=178.2(\mathrm{C}=\mathrm{O}), 157.2(1-\mathrm{C}, 8-$ C), 153.0 (4a-C, 4b-C), 128.0 (3-C, 6-C), 112.2 (8a-C, 9a-C), 109.1 (4-C, 5-C), 104.5 (2-C, 7-C), $55.4\left(\mathrm{OCH}_{3}\right), 40.6\left(\mathrm{CH}_{2} \mathrm{CO}_{2} \mathrm{H}\right), 26.1$ (9-C).

HRMS (ESI): $m / z$ [M+ Na] $]^{+}$calcd. for $\mathrm{C}_{17} \mathrm{H}_{16} \mathrm{NaO}_{5}$ : 323.08954; found: 323.08899 .

Hydrolysis of diethoxy aryl ethyl ester. Preparation of (1,8-diethoxy2,3,6,7-tetramethyl-9H-xanthen-9-yl)-acetic acid (40). Aqueous $\mathrm{KOH}$ solution $(5.0 \mathrm{M}, 1.6 \mathrm{~mL}, 8 \mathrm{mmol})$ was added to a solution of ethyl ester $(0.24 \mathrm{mmol})$ in $\mathrm{EtOH}(4.0 \mathrm{~mL})$. The reaction mixture was refluxed under stirring for 2 hours. After removal of $\mathrm{EtOH}$ in vacuo, the residue was redissolved with $\mathrm{EtAcO}$. The aqueous layers were cautiously acidified to $\mathrm{pH}=4$ with $10 \% \mathrm{HCl}$ and extracted with EtAcO $(3 \times 5 \mathrm{~mL})$. The combined organic layers were dried over anhydrous sodium sulphate. After filtration and concentration, the residue was purified by silica gel column chromatography.

Yield: quantitative, White solid, Mp: $206.4-207.0^{\circ} \mathrm{C}$, Hex:EtOAc 90 : 10. 
${ }^{1} \mathrm{H} \mathrm{NMR}\left(300 \mathrm{MHz}, \mathrm{CDCl}_{3}, 25^{\circ} \mathrm{C}, \mathrm{TMS}\right): \delta=6.39$ (s, 2H, 4-ArH), 4.83 (t, $J$ $=5.5 \mathrm{~Hz}, 1 \mathrm{H}, \mathrm{CHCH}_{2} \mathrm{CO}_{2} \mathrm{H}$ ), 4.01 (d quartet, $J=2.1,7.1 \mathrm{~Hz}, 4 \mathrm{H}$, $\left.\mathrm{OCH}_{2} \mathrm{CH}_{3}\right), 2.68\left(\mathrm{~d}, J=5.5 \mathrm{~Hz}, 2 \mathrm{H}, \mathrm{CH}_{2} \mathrm{CO}_{2} \mathrm{H}\right), 2.27\left(\mathrm{~s}, 6 \mathrm{H}, 3,6-\mathrm{CH}_{3}\right)$, $2.24\left(\mathrm{~s}, 6 \mathrm{H}, 2,7-\mathrm{CH}_{3}\right), 1.40\left(\mathrm{t}, J=7.1 \mathrm{~Hz}, 6 \mathrm{H}, \mathrm{OCH}_{2} \mathrm{CH}_{3}\right)$.

${ }^{13} \mathrm{C}$ NMR $\left(75 \mathrm{MHz}, \mathrm{CDCl}_{3}, 25^{\circ} \mathrm{C}, \mathrm{TMS}\right): \delta=177.5(\mathrm{C}=\mathrm{O}), 151.2(1-\mathrm{C}, 8-$ C), 153.6 (4a-C, 4b-C), 135.9 (2-C, 7-C), 116.7 (3-C, 6-C), 110.0 (8a-C, 9a-C), 106.8 (4-C, 5-C), $63.5\left(\mathrm{OCH}_{2} \mathrm{CH}_{3}\right), 41.5\left(\mathrm{CH}_{2} \mathrm{CO}_{2} \mathrm{H}\right), 26.2(9-\mathrm{C})$, $20.3\left(3,6-\mathrm{CH}_{3}\right), 14.7\left(\mathrm{OCH}_{2} \mathrm{CH}_{3}\right), 11.3\left(2,7-\mathrm{CH}_{3}\right)$.

HRMS (ESI): $m / z$ [M+ 2Na] ${ }^{+}$calcd. for $\mathrm{C}_{23} \mathrm{H}_{27} \mathrm{Na}_{2} \mathrm{O}_{5}:$ 429.16484; found: 429.16355 .

\section{N-Benzyl-2-(1,8-dioxo-2,3,4,5,6,7,8,9-octahydro-1H-xanthen-9-yl)- acetamide (41).}

Yield 71.3\%. $\mathrm{CH}_{2} \mathrm{Cl}_{2}: \mathrm{MeOH}$ 94: 6. Yellow oil.

IR (film): $3190(\mathrm{~N}-\mathrm{H}), 2949(\mathrm{CH}), 1674(\mathrm{C}=\mathrm{O}), 1647$ (C=O amide), 1616 $(\mathrm{C}=\mathrm{C}), 1543(\mathrm{C}-\mathrm{N}), 1381\left(\mathrm{CH}_{2}\right), 1176(\mathrm{C}-\mathrm{O}) \mathrm{cm}^{-1}$.

${ }^{1} \mathrm{H} \mathrm{NMR}\left(300 \mathrm{MHz}, \mathrm{CDCl}_{3}, 25^{\circ} \mathrm{C}, \mathrm{TMS}\right): \delta=7.36-7.25(\mathrm{~m}, 5 \mathrm{H}, \mathrm{Ar}-\mathrm{H})$, 5.98 (bs, $1 \mathrm{H}, \mathrm{NH}$ ), 4.34 (d, $\left.2 \mathrm{H}, J=5.8 \mathrm{~Hz}, \mathrm{CH}_{2} \mathrm{Ar}\right), 3.89(\mathrm{t}, 1 \mathrm{H}, J=4.4 \mathrm{~Hz}$ 9-H), 2.49-2.37 (m, 8H, 2-H, 4-H, 5-H, 7-H), $2.48(\mathrm{~d}, 2 \mathrm{H}, J=4.4 \mathrm{~Hz}$, $\left.\mathrm{CH}_{2} \mathrm{C}(\mathrm{O}) \mathrm{NH}\right), 2.03-1.90(\mathrm{~m}, 4 \mathrm{H}, 3-\mathrm{H}, 6-\mathrm{H})$.

${ }^{13} \mathrm{C} \mathrm{NMR}\left(75 \mathrm{MHz}, \mathrm{CDCl}_{3}, 25^{\circ} \mathrm{C}, \mathrm{TMS}\right): \delta=197.8$ (1-C, 8-C), 171.2 (CONH), 165.9 (4a-C, 4b-C), 138.6, 128.6, 128.0, 127.3 (Ar), 114.8 (8aC, 9a-C), $43.4\left(\mathrm{CH}_{2} \mathrm{Ar}\right), 40.3\left(\mathrm{CH}_{2} \mathrm{C}(\mathrm{O}) \mathrm{NH}\right), 36.9(2-\mathrm{C}, 7-\mathrm{C}), 27.1(4-\mathrm{C}, 5-$ C), 23.9 (9-C), 20.3 (3-C, 6-C).

HRMS (ESI): $m / z$ [M+ Na] ${ }^{+}$calcd. for $\mathrm{C}_{22} \mathrm{H}_{23} \mathrm{NNaO}_{4}:$ 388.15193; found: 388.15089 .

\section{N-Benzyl-2-(3,6-dimethyl-1,8-dioxo-2,3,4,5,6,7,8,9-octahydro-1H-} xanthen-9-yl)-acetamide (42).

Yield $60 \%$.. Hex:EtOAc 45 : 55. White solid. Mp: $140.2-140.6^{\circ} \mathrm{C}$.

IR (film): $3440(\mathrm{~N}-\mathrm{H}), 2953(\mathrm{CH}), 1647$ (C=O), $1630(\mathrm{C}=\mathrm{C}), 1456(\mathrm{C}-\mathrm{N})$ $1382\left(\mathrm{CH}\right.$ of methyl), $1190,1132 \mathrm{~cm}^{-1}$.

${ }^{1} \mathrm{H}$ NMR $\left(300 \mathrm{MHz}, \mathrm{CDCl}_{3}, 25^{\circ} \mathrm{C}, \mathrm{TMS}\right): \delta=7.27-7.25(\mathrm{~m}, 5 \mathrm{H}, \mathrm{Ar}-\mathrm{H})$ 6.22 (br.s, $1 \mathrm{H}, \mathrm{NH}$ ), 4.30 (d, $J=5.7 \mathrm{~Hz}, 2 \mathrm{H}, \mathrm{CH}_{2} \mathrm{Ar}$ ), 3.83 (br.s, $1 \mathrm{H}, 9-\mathrm{H}$ ) 2.55-2.31 (m, 8H, 2- H, 4- H, 5- H, 7- $), 2.31-1.83(\mathrm{~m}, 4 \mathrm{H}, 3-\mathrm{H}, 6-\mathrm{H}$, $\left.\mathrm{CH}_{2} \mathrm{CONH}\right), 1.05\left(\mathrm{~d}, 3 \mathrm{H}, J=5.4 \mathrm{~Hz}, \mathrm{CH}_{3}\right), 1.02\left(\mathrm{~d}, 3 \mathrm{H}, J=5.3 \mathrm{~Hz}, \mathrm{CH}_{3}\right)$.

${ }^{13} \mathrm{C}$ NMR $\left(75 \mathrm{MHz}, \mathrm{CDCl} 3,25^{\circ} \mathrm{C}, \mathrm{TMS}\right): \delta=197.7$ (1-C, 8-C), 171.2 $(\mathrm{C}(\mathrm{O}) \mathrm{NH}), 165.8(4 \mathrm{a}-\mathrm{C}, 4 \mathrm{~b}-\mathrm{C}), 138.7,128.6,128.1,127.3(\mathrm{Ar}), 114.4(8 \mathrm{a}-$ C, 9a-C), $45.3(2-\mathrm{C}, 7-\mathrm{C}), 43.3\left(\mathrm{CH}_{2} \mathrm{Ar}\right), 40.4\left(\mathrm{CH}_{2} \mathrm{CONH}\right), 35.3$ (4-C, 5C), 28.3, 27.9 (3-C, 6-C), $24.4(9-\mathrm{C}), 20.7\left(\mathrm{CH}_{3}\right)$.

HRMS (ESI): $\mathrm{m} / \mathrm{z}$ [M+ Na] ${ }^{+}$calcd. for $\mathrm{C}_{24} \mathrm{H}_{27} \mathrm{NNaO}_{4}: 416.18323$; found: 416.18455 .

\section{N-Benzyl-2-(3,3,6,6-tetramethyl-1,8-dioxo-2,3,4,5,6,7,8,9-octahydro-} $1 H$-xanthen-9-yl)-acetamide (43).

Yield 53 \%. Hex:EtOAc 57: 43, White crystals. Mp: 195.2-195.5 ${ }^{\circ} \mathrm{C}$.

IR (film): $3321(\mathrm{~N}-\mathrm{H}), 2959(\mathrm{CH}), 1673$ (C=O), 1662 (C=O amide), 1620 $(\mathrm{C}=\mathrm{C}), 1539(\mathrm{C}-\mathrm{N}), 1380\left(\mathrm{C}-\mathrm{N}, \mathrm{gemCH} \mathrm{Cm}_{3} \mathrm{~cm}^{-1}\right.$.

${ }^{1} \mathrm{H}$ NMR $\left(300 \mathrm{MHz}, \mathrm{CDCl}_{3}, 25^{\circ} \mathrm{C}, \mathrm{TMS}\right): \delta=7.31-7.21(\mathrm{~m}, 5 \mathrm{H}, \mathrm{Ar}-\mathrm{H})$, 5.82 (bs, $1 \mathrm{H}, \mathrm{NH}$ ), 4.30 (d, $J=5.7 \mathrm{~Hz}, 2 \mathrm{H}, \mathrm{CH}_{2} \mathrm{Ar}$ ), 3.85 (t, $J=3.9 \mathrm{~Hz}, 1 \mathrm{H}$ 9-H), $2.57\left(\mathrm{~d}, J=4.3 \mathrm{~Hz}, 2 \mathrm{H}, \mathrm{CH}_{2} \mathrm{C}(\mathrm{O}) \mathrm{NH}\right), 2.37(\mathrm{~d}, J=17.7 \mathrm{~Hz}, 4 \mathrm{H}, 2-\mathrm{H}$,
7-H), $2.24(\mathrm{~d}, J=17.7 \mathrm{~Hz}, 4 \mathrm{H}, 4-\mathrm{H}, 5-\mathrm{H}), 1.11\left(\mathrm{~s}, 6 \mathrm{H}, \mathrm{CH}_{3}\right), 1.09(\mathrm{~s}, 6 \mathrm{H}$ $\left.\mathrm{CH}_{3}\right)$.

${ }^{13} \mathrm{C}$ NMR $\left(75 \mathrm{MHz}, \mathrm{CDCl}_{3}, 25^{\circ} \mathrm{C}, \mathrm{TMS}\right): \delta=197.7$ (1-C, 8-C), 171.2 $(\mathrm{C}(\mathrm{O}) \mathrm{NH}), 164.6$ (4a-C, 4b-C), 138.4, 128.6, 128.0, 127.4 (Ar), 113.5 (8aC, 9a-C), 50.8 (2-C, 7-C), $43.4\left(\mathrm{CH}_{2} \mathrm{Ar}\right), 40.8$ (4-C, 5-C), 38.9 $\left(\mathrm{CH}_{2} \mathrm{C}(\mathrm{O}) \mathrm{NH}\right), 32.1(3-\mathrm{C}, 6-\mathrm{C}), 29.1\left(\mathrm{CH}_{3}\right), 27.3\left(\mathrm{CH}_{3}\right), 24.3(9-\mathrm{C})$.

HRMS (ESI): $\mathrm{m} / \mathrm{z}[\mathrm{M}+\mathrm{K}]^{+}$calcd. for $\mathrm{C}_{26} \mathrm{H}_{31} \mathrm{KNO}_{4}:$ : 460.18847; found: 460.18708 .

$N$-Benzyl-2-(2,2,5,5-tetramethyl-1,8-dioxo-2,3,4,5,6,7,8,9-octahydro$1 H$-xanthen-9-yl)-acetamide (44).

Yield 54 \%. Hex: EtOAc 62: 38. Yellow oil.

IR (film): $3341(\mathrm{~N}-\mathrm{H}), 2963,2926(\mathrm{CH}), 1674,1645$ (C=O amide, C=O ketone, Ar), $1620(\mathrm{C}=\mathrm{C}), 1381(\mathrm{C}-\mathrm{N}), 1175(\mathrm{C}-\mathrm{O}) \mathrm{cm}^{-1}$.

${ }^{1} \mathrm{H}$ NMR $\left(300 \mathrm{MHz}, \mathrm{CDCl}_{3}, 25^{\circ} \mathrm{C}, \mathrm{TMS}\right): \delta=7.33-7.24(\mathrm{~m}, 5 \mathrm{H}, \mathrm{Ar}-\mathrm{H})$, 5.95 (bs, $1 \mathrm{H}, \mathrm{NH}$ ), 4.40 (dd, $J=15.0,6.0 \mathrm{~Hz}, 1 \mathrm{H}, \mathrm{CH}_{2} \mathrm{Ar}$ ), 4.26 (dd, $J=$ $\left.15.0,6.0 \mathrm{~Hz}, 1 \mathrm{H}, \mathrm{CH}_{2} \mathrm{Ar}\right), 3.87(\mathrm{t}, J=4.1 \mathrm{~Hz}, 1 \mathrm{H}, 9-\mathrm{H}), 2.57-2.40(\mathrm{~m}, 6 \mathrm{H}$, $\left.\mathrm{CH}_{2} \mathrm{C}(\mathrm{O}) \mathrm{NH}, 4-\mathrm{H}, 7-\mathrm{H}\right), 1.89-1.76(\mathrm{~m}, 4 \mathrm{H}, 3-\mathrm{H}, 6-\mathrm{H}), 1.30\left(\mathrm{~s}, 3 \mathrm{H}, 5-\mathrm{CH}_{3}\right)$, $1.22\left(\mathrm{~s}, 3 \mathrm{H}, 5-\mathrm{CH}_{3}\right), 1.10\left(\mathrm{~s}, 3 \mathrm{H}, 2-\mathrm{CH}_{3}\right), 1.08\left(\mathrm{~s}, 3 \mathrm{H}, 2-\mathrm{CH}_{3}\right)$.

${ }^{13} \mathrm{C}$ NMR $\left(75 \mathrm{MHz}, \mathrm{CDCl}_{3}, 25^{\circ} \mathrm{C}, \mathrm{TMS}\right): \delta=202.4$ (1-C), 197.5 (8-C), $171.0(\mathrm{C}(\mathrm{O}) \mathrm{NH}), 170.8$ (4b-C), $164.1(4 \mathrm{a}-\mathrm{C}), 138.5,128.6,127.9,127.4$ (Ar), 113.1, $112.7(8 \mathrm{a}-\mathrm{C}, 9 \mathrm{a}-\mathrm{C}), 43.5\left(\mathrm{CH}_{2} \mathrm{Ar}\right), 40.7\left(\mathrm{CH}_{2} \mathrm{C}(\mathrm{O}) \mathrm{NH}\right), 40.5$ (2-C), 33.8 (7-C), 35.3 and $34.0(3-\mathrm{C}, 6-\mathrm{C}), 34.5(5-\mathrm{C}), 26.2\left(5-\mathrm{CH}_{3}\right), 24.7$ $\left(2-\mathrm{CH}_{3}\right), 24.5(9-\mathrm{C}), 24.4\left(5-\mathrm{CH}_{3}\right), 24.2\left(2-\mathrm{CH}_{3}\right), 24.0(4-\mathrm{C})$.

HRMS (ESI): $m / z$ [M+ Na] ${ }^{+}$calcd. for $\mathrm{C}_{26} \mathrm{H}_{31} \mathrm{NNaO}_{4}: 444.21453$; found 444.21390 .

N-(3-Dimethylamino-propyl)-2-(1,8-dioxo-2,3,4,5,6,7,8,9-octahydro$1 H$-xanthen-9-yl)-acetamide (45).

Yield $99 \% . \mathrm{MeOH}: \mathrm{NH}_{3}$ 95: 5. Yellow oil.

IR (film): $3363(\mathrm{~N}-\mathrm{H}), 2947(\mathrm{CH}), 1658(\mathrm{C}=\mathrm{O}), 1651$ (C=O amide), 1554 (C-N), $1265(\mathrm{C}-\mathrm{N}) \mathrm{cm}^{-1}$

$\left.{ }^{1} \mathrm{H} \mathrm{NMR} \mathrm{(300} \mathrm{MHz,} \mathrm{CDCl}_{3}, 25^{\circ} \mathrm{C}, \mathrm{TMS}\right): \delta=6.85$ (br.s, $\left.1 \mathrm{H}, \mathrm{NH}\right), 3.85$ (t, $J$ $=4.6 \mathrm{~Hz}, 1 \mathrm{H}, 9-\mathrm{H}), 3.21\left(\mathrm{q}, \mathrm{J}=6.2 \mathrm{~Hz}, 2 \mathrm{H}, \mathrm{NHCH}_{2}\right), 2.63-2.27(\mathrm{~m}, 12 \mathrm{H}$, $\left.2-\mathrm{H}, 4-\mathrm{H}, 5-\mathrm{H}, 7-\mathrm{H}, \mathrm{CH}_{2} \mathrm{CONH}, \mathrm{CH}_{2} \mathrm{~N}\left(\mathrm{CH}_{3}\right)_{2}\right), 2.34\left(\mathrm{~s}, 6 \mathrm{H}, \mathrm{N}\left(\mathrm{CH}_{3}\right)_{2}\right), 2.07-$ $1.99(\mathrm{~m}, 4 \mathrm{H}, 3-\mathrm{H}, 6-\mathrm{H}), 1.70\left(\mathrm{~m}, 2 \mathrm{H}, \mathrm{CH}_{2} \mathrm{CH}_{2} \mathrm{~N}\left(\mathrm{CH}_{3}\right)_{2}\right)$.

${ }^{13} \mathrm{C}$ NMR $\left(75 \mathrm{MHz}, \mathrm{CDCl}_{3}, 25^{\circ} \mathrm{C}, \mathrm{TMS}\right): \delta=197.6(1-\mathrm{C}, 8-\mathrm{C}), 171.5$ (CONH), 165.8 (4a-C, 4b-C), $115.2(8 \mathrm{a}-\mathrm{C}, 9 \mathrm{a}-\mathrm{C}), 57.3\left(\mathrm{CH}_{2} \mathrm{~N}\left(\mathrm{CH}_{3}\right)_{2}\right)$,

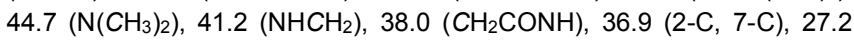
(4-C, 5-C), $26.2\left(\mathrm{CH}_{2} \mathrm{CH}_{2} \mathrm{~N}\left(\mathrm{CH}_{3}\right)_{2}\right), 24.1$ (9-C), 20.4 (3-C, 6-C).

HRMS (ESI): $m / z$ [M+ Na] ${ }^{+}$calcd. for $\mathrm{C}_{20} \mathrm{H}_{28} \mathrm{~N}_{2} \mathrm{NaO}_{4}: 383.19413$; found: 383.19337 .

$N$-(3-Dimethylamino-propyl)-2-(3,6-dimethyl-1,8-dioxo-2,3,4,5,6,7,8,9octahydro-1H-xanthen-9-yl)-acetamide (46).

Yield: $68 \% . \mathrm{CH}_{2} \mathrm{Cl}_{2}: \mathrm{MeOH} 75: 25$. Pale yellow oil.

IR (film): $3381(\mathrm{~N}-\mathrm{H}), 2953(\mathrm{CH}), 1645(\mathrm{C}=\mathrm{O}), 1614(\mathrm{C}=\mathrm{C}), 1450(\mathrm{C}-\mathrm{N})$, 1385 (CH methyl), 1190, 1099, $744 \mathrm{~cm}^{-1}$.

${ }^{1} \mathrm{H}$ NMR $\left(\mathrm{D}_{2} \mathrm{O}, 300 \mathrm{MHz}\right): \delta=3.42(\mathrm{t}, J=4.5 \mathrm{~Hz}, 1 \mathrm{H}, 9-\mathrm{H}), 2.92(\mathrm{t}, \quad J=7$ $\left.\mathrm{Hz}, 2 \mathrm{H}, \mathrm{NHCH}_{2}\right), 2.76\left(\mathrm{t}, J=7 \mathrm{~Hz}, 2 \mathrm{H}, \mathrm{CH}_{2} \mathrm{~N}\left(\mathrm{CH}_{3}\right)_{2}\right), 2.56\left(\mathrm{~s}, 6 \mathrm{H}, \mathrm{N}-\mathrm{CH}_{3}\right)$, 
2.40-2.25 (m, 6H, 4- H, 5- $\left.\mathrm{H}, \mathrm{CH}_{2} \mathrm{C}(\mathrm{O}) \mathrm{NH}\right)$, 2.25-2.00 (m, 6H, 2-H, 3- $\mathrm{H}, 6-$ $\mathrm{H}, 7-\mathrm{H}), 1.62\left(\mathrm{~m}, 2 \mathrm{H}, \mathrm{CH}_{2} \mathrm{CH}_{2} \mathrm{~N}\left(\mathrm{CH}_{3}\right)_{2}\right), 1.02-0.89\left(\mathrm{~m}, 6 \mathrm{H}, \mathrm{CH}_{3}\right)$

${ }^{13} \mathrm{C} \mathrm{NMR}\left(75 \mathrm{MHz}, \mathrm{CDCl}_{3}, 25^{\circ} \mathrm{C}, \mathrm{TMS}\right): \delta=201.7(1-\mathrm{C}, 8-\mathrm{C}), 173.5$ (C(O)NH), 168.7 (4a-C, 4b-C), $113.5(8 \mathrm{a}-\mathrm{C}, 9 \mathrm{a}-\mathrm{C}), 55.3\left(\mathrm{CH}_{2} \mathrm{~N}-g e m \mathrm{CH}_{3}\right)$, $42.9\left(\mathrm{~N}-g e m \mathrm{CH}_{3}\right), \quad 39.6 \quad\left(\mathrm{CH}_{2} \mathrm{C}(\mathrm{O}) \mathrm{NH}\right), \quad 38.5 \quad(2-\mathrm{C}, \quad 7-\mathrm{C}), \quad 36.3$ $\left(\mathrm{C}(\mathrm{O}) \mathrm{NHCH}_{2}\right), 34.4$ (4-C, 5-C), 27.4 (3-C, 6-C), $24.4\left(\mathrm{CH}_{2} \mathrm{CH}_{2} \mathrm{CH}_{2} \mathrm{NMe}_{2}\right)$ $23.5(9-\mathrm{C}), 21.5\left(\mathrm{CH}_{3}\right), 19.7\left(\mathrm{CH}_{3}\right)$.

HRMS (ESI): $m / z[M+H]^{+}$calcd. for $\mathrm{C}_{22} \mathrm{H}_{33} \mathrm{~N}_{2} \mathrm{O}_{4}$ : 389.24348; found: 389.24296 .

\section{N-(3-Dimethylamino-propyl)-2-(3,3,6,6-tetramethyl-1,8-dioxo- 2,3,4,5,6,7,8,9-octahydro-1H-xanthen-9-yl)-acetamide (47)}

Yield: $88.2 \%$. $\mathrm{CH}_{2} \mathrm{Cl}_{2}: \mathrm{MeOH}$ 80: 20. Colourless oil.

IR (film): $3357(\mathrm{~N}-\mathrm{H}), 2956(\mathrm{CH}), 1661(\mathrm{C}=\mathrm{O}), 1654$ (C=O amide), 1620 $(\mathrm{C}=\mathrm{C}), 1382(\mathrm{C}-\mathrm{N}), 1380(\mathrm{gemCH}) \mathrm{cm}^{-1}$.

${ }^{1} \mathrm{H}$ NMR $\left(300 \mathrm{MHz}, \mathrm{CDCl}_{3}, 25^{\circ} \mathrm{C}, \mathrm{TMS}\right): \delta=6.68$ (br.s, $\left.1 \mathrm{H}, \mathrm{NH}\right), 3.82(\mathrm{t}$, $J=3.8 \mathrm{~Hz}, 1 \mathrm{H}, 9-\mathrm{H}), 3.17$ (q, J=6.4 Hz, 2H, $\left.\mathrm{NHCH}_{2}\right), 2.48$ (d, $J=4.3 \mathrm{~Hz}$ $2 \mathrm{H}, \mathrm{CH}_{2} \mathrm{CONH}$ ), $2.30\left(\mathrm{t}, \mathrm{J}=6.4 \mathrm{~Hz}, 2 \mathrm{H}, \mathrm{CH}_{2} \mathrm{~N}\left(\mathrm{CH}_{3}\right)_{2}\right), 2.20(\mathrm{~s}, 6 \mathrm{H}$, $\left.\mathrm{N}\left(\mathrm{CH}_{3}\right)_{2}\right), 2.38(\mathrm{~s}, 4 \mathrm{H}, 2-\mathrm{H}, 7-\mathrm{H}), 2.28(\mathrm{~s}, 4 \mathrm{H}, 4-\mathrm{H}, 5-\mathrm{H}), 1.57(\mathrm{~m}, 2 \mathrm{H}$, $\left.\mathrm{CH}_{2} \mathrm{CH}_{2} \mathrm{~N}\left(\mathrm{CH}_{3}\right)_{2}\right), 1.12$ and $1.09\left(2 \mathrm{~s}, 12 \mathrm{H}, \mathrm{CH}_{3}\right)$.

${ }^{13} \mathrm{C}$ NMR $\left(75 \mathrm{MHz}, \mathrm{CDCl}_{3}, 25^{\circ} \mathrm{C}, \mathrm{TMS}\right): \delta=197.6(1-\mathrm{C}, 8-\mathrm{C}), 171.4$ $(\mathrm{C}(\mathrm{O}) \mathrm{NH}), 164.6$ (4a-C, 4b-C), 113.7 (8a-C, 9a-C), $58.3\left(\mathrm{CH}_{2} \mathrm{~N}\left(\mathrm{CH}_{3}\right)_{2}\right)$, 51.0 (2-C, 7-C), $45.4\left(\mathrm{~N}\left(\mathrm{CH}_{3}\right)_{2}\right), 40.9(4-\mathrm{C}, 5-\mathrm{C}), 39.0\left(\mathrm{NHCH}_{2}\right), 38.9$ $\left(\mathrm{CH}_{2} \mathrm{CONH}\right), \quad 32.1 \quad\left(\mathrm{C}-\mathrm{gemCH} \mathrm{CH}_{3}\right), \quad 29.4 \quad\left(\mathrm{CH}_{3}\right), \quad 27.1 \quad\left(\mathrm{CH}_{3}\right), \quad 26.3$ $\left(\mathrm{CH}_{2} \mathrm{CH}_{2} \mathrm{~N}\left(\mathrm{CH}_{3}\right)_{2}\right), 24.2$ (9-C).

HRMS (ESI): $m / z$ [M+ Na] ${ }^{+}$calcd. for $\mathrm{C}_{24} \mathrm{H}_{36} \mathrm{~N}_{2} \mathrm{NaO}_{4}$ : 439.25673; found: 439.25571.

\section{N-(3-Dimethylamino-propyl)-2-(2,2,5,5-tetramethyl-1,8-dioxo- 2,3,4,5,6,7,8,9-octahydro-1H-xanthen-9-yl)-acetamide (48).}

Yield 58\%. $\mathrm{CH}_{2} \mathrm{Cl}_{2}: \mathrm{MeOH}$ 40: 60. Yellow oil.

IR (film): $3362(\mathrm{~N}-\mathrm{H}), 2963(\mathrm{CH}), 1651(\mathrm{C}=\mathrm{O}), 1665$ (C=O amide), 1614 $(\mathrm{C}=\mathrm{C}), 1379(\mathrm{C}-\mathrm{N}), 1174 \mathrm{~cm}^{-1}$.

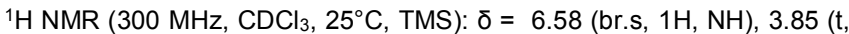
$J=4.6 \mathrm{~Hz}, 1 \mathrm{H}, 9-\mathrm{H}$ ), 3.19 (ddd, $J=13.5,6.4,3.0 \mathrm{~Hz}, 2 \mathrm{H}, \mathrm{NHCH}_{2}$ ), 2.54 (m, $\left.2 \mathrm{H}, \mathrm{CH}_{2} \mathrm{~N}\left(\mathrm{CH}_{3}\right)_{2}\right), 2.45(\mathrm{t}, \mathrm{J}=6.4 \mathrm{~Hz}, 2 \mathrm{H}, 7-\mathrm{H}), 2.33-2.28(\mathrm{~m}, 4 \mathrm{H}, 4-\mathrm{H}$, $\left.\mathrm{CH}_{2} \mathrm{C}(\mathrm{O}) \mathrm{NH}\right), 2.19\left(\mathrm{~s}, 6 \mathrm{H}, \mathrm{N}\left(\mathrm{CH}_{3}\right)_{2}\right), 1.94-1.78(\mathrm{~m}, 4 \mathrm{H}, 3-\mathrm{H}, 6-\mathrm{H}), 1.60$ (q, $\left.J=6.7 \mathrm{~Hz}, 2 \mathrm{H}, \mathrm{CH}_{2} \mathrm{CH}_{2} \mathrm{~N}\left(\mathrm{CH}_{3}\right)_{2}\right), 1.29\left(\mathrm{~s}, 3 \mathrm{H}, 5-\mathrm{CH}_{3}\right), 1.22\left(\mathrm{~s}, 3 \mathrm{H}, 5-\mathrm{CH}_{3}\right)$ $1.15\left(\mathrm{~s}, 3 \mathrm{H}, 2-\mathrm{CH}_{3}\right), 1.09\left(\mathrm{~s}, 3 \mathrm{H}, 2-\mathrm{CH}_{3}\right)$.

${ }^{13} \mathrm{C}$ NMR $\left(75 \mathrm{MHz}, \mathrm{CDCl}_{3}, 25^{\circ} \mathrm{C}, \mathrm{TMS}\right): \delta=202.3(1-\mathrm{C}), 196.8$ (8-C), $171.1(\mathrm{C}(\mathrm{O}) \mathrm{NH}), 170.9(4 \mathrm{~b}-\mathrm{C}), 163.9(4 \mathrm{a}-\mathrm{C}), 113.1$ and 113.0 (8a-C, 9aC), $58.1\left(\mathrm{CH}_{2} \mathrm{~N}\left(\mathrm{CH}_{3}\right)_{2}\right), 45.4\left(\mathrm{~N}\left(\mathrm{CH}_{3}\right)_{2}\right), 40.8\left(\mathrm{CH}_{2} \mathrm{C}(\mathrm{O}) \mathrm{NH}\right), 40.5(2-\mathrm{C})$, $38.4\left(\mathrm{NHCH}_{2}\right), 35.4$ (6-C), 34.5 (5-C), 34.1 (3-C), 33.8 (7-C), 26.7 $\left(\mathrm{CH}_{2} \mathrm{CH}_{2} \mathrm{~N}\left(\mathrm{CH}_{3}\right)_{2}\right), 26.3\left(5-\mathrm{CH}_{3}\right), 24.7\left(2-\mathrm{CH}_{3}\right), 24.4(9-\mathrm{C}), 24.4\left(5-\mathrm{CH}_{3}\right)$, $24.2\left(2-\mathrm{CH}_{3}\right), 24.0(4-\mathrm{C})$.

HRMS (ESI): $m / z[M+H]^{+}$calcd. for $\mathrm{C}_{24} \mathrm{H}_{37} \mathrm{~N}_{2} \mathrm{O}_{4}$ : 417.27478; found: 417.27359.

\section{2-(1,8-Dimethoxy-9H-xanthen-9-yl)-N-(3-dimethylamino-propyl)-} acetamide (49).

Yield: $60 \% . \mathrm{CH}_{2} \mathrm{Cl}_{2}$ : $\mathrm{MeOH} 88$ : 12. Colourless oil.
IR (film) 3300 (N-H), 2934 (CH), 2837 (OMe), 1651 (C=O amide), 1620 $(\mathrm{C}=\mathrm{CAr}), 1456(\mathrm{Ar}), 1289\left(\mathrm{ArOCH}_{3}\right), 1094(\mathrm{C}-\mathrm{N}-\mathrm{C}), 1082 \mathrm{~cm}^{-1}$.

${ }^{1} \mathrm{H}$ NMR $\left(300 \mathrm{MHz}, \mathrm{CDCl}_{3}, 25^{\circ} \mathrm{C}, \mathrm{TMS}\right): \delta=7.15(\mathrm{t}, \mathrm{J}=8.3 \mathrm{~Hz}, 2 \mathrm{H}, 3$ $\mathrm{ArH}, 6-\mathrm{ArH}), 6.69$ (d, J = 8.0 Hz, 2H, 2-ArH, 7-ArH), 6.59 (d, J = 8.0 Hz, $2 \mathrm{H}, 4-\mathrm{ArH}, 5-\mathrm{ArH}), 5.89(\mathrm{br}, \mathrm{s}, 1 \mathrm{H}, \mathrm{NH}), 4.81(\mathrm{t}, J=5.4 \mathrm{~Hz}, 1 \mathrm{H}, 9-\mathrm{H}), 3.87$ (s, $\left.6 \mathrm{H}, \mathrm{OCH}_{3}\right), 3.10\left(\mathrm{~m}, 2 \mathrm{H}, \mathrm{CH}_{2} \mathrm{NHC}(\mathrm{O})\right), 2.58\left(\mathrm{~d}, 2 \mathrm{H}, \mathrm{CH}_{2} \mathrm{CONH}\right), 2.23$ $\left(\mathrm{m}, \quad 2 \mathrm{H}, \quad \mathrm{CH}_{2} \mathrm{~N}\left(\mathrm{CH}_{3}\right)_{2}\right), 2.19\left(\mathrm{~s}, \quad 6 \mathrm{H}, \quad \mathrm{N}\left(\mathrm{CH}_{3}\right)_{2}\right), \quad 1.48 \quad(\mathrm{~m}, 2 \mathrm{H}$, $\left.\mathrm{CH}_{2} \mathrm{CH}_{2} \mathrm{NHC}(\mathrm{O})\right)$.

${ }^{13} \mathrm{C}$ NMR $\left(75 \mathrm{MHz}, \mathrm{CDCl}_{3}, 25^{\circ} \mathrm{C}, \mathrm{TMS}\right): \delta=171.0(\mathrm{C}(\mathrm{O}) \mathrm{NH}), 157.1(1-\mathrm{C}$, 8-C), 153.1 (4a-C, 4b-C), 127.9 (3-C, 6-C), 113.1 (8a-C, 9a-C), 109.1 (4C, 5-C), 104.7 (2-C, 7-C), $57.5\left(\left(\mathrm{CH}_{3}\right)_{2} \mathrm{NCH}_{2} \mathrm{CH}_{2} \mathrm{CH}_{2} \mathrm{NH}-\mathrm{C}=\mathrm{O}\right), 45.1$ $\left(\left(\mathrm{CH}_{3}\right)_{2} \mathrm{~N}-\right), 43.3\left(\left(\mathrm{CH}_{3}\right)_{2} \mathrm{NCH}_{2} \mathrm{CH}_{2} \mathrm{CH}_{2} \mathrm{NH}-\mathrm{C}=\mathrm{O}\right), 38.2\left(\mathrm{CH}_{2} \mathrm{C}(\mathrm{O}) \mathrm{N}\right), 26.6$ $\left(\left(\mathrm{CH}_{3}\right)_{2} \mathrm{NCH}_{2} \mathrm{CH}_{2} \mathrm{CH}_{2} \mathrm{NH}-\mathrm{C}=\mathrm{O}\right), 26.4$ (9-C).

HRMS (ESI): $\mathrm{m} / \mathrm{z}[\mathrm{M}+\mathrm{H}]^{+}$calcd. for $\mathrm{C}_{22} \mathrm{H}_{29} \mathrm{~N}_{2} \mathrm{O}_{4}$ : 385.21088; found: 385.21218

2-(1,8-Diethoxy-2,3,6,7-tetramethyl-9H-xanthen-9-yl)-N-(3dimethylamino-propyl)-acetamide (50).

Yield: $51 \% . \mathrm{CH}_{2} \mathrm{Cl}_{2}: \mathrm{MeOH}$ 96: 4. Colourless oil.

IR (film): $3340(\mathrm{~N}-\mathrm{H}), 2972(=\mathrm{CH}), 2926(\mathrm{CH}), 2874,1633$ (C=O amide), 1575 (C=CAr), 1506, 1450 (Ar), 1202 (C-O-CAr), 1168, $1107(\mathrm{C}-\mathrm{N}-\mathrm{C}) \mathrm{cm}^{-}$ 1

${ }^{1} \mathrm{H}$ NMR $\left(300 \mathrm{MHz}, \mathrm{CDCl}_{3}, 25^{\circ} \mathrm{C}, \mathrm{TMS}\right): \delta=6.43(\mathrm{~s}, 1 \mathrm{H}, 4-\mathrm{Ar}-\mathrm{H}), 5.90(\mathrm{t}$ $J=6.0 \mathrm{~Hz}, 1 \mathrm{H}, \mathrm{NH}), 4.74(\mathrm{t}, J=5.1,1 \mathrm{H}, 9-\mathrm{H}), 4.08\left(\mathrm{ABX}_{3}\right.$ system, $J_{A B}=$ 14.2, $\left.J_{A X}=J_{B X}=7.1 \mathrm{~Hz}, 2 \mathrm{H}, \mathrm{OCH}_{2} \mathrm{CH}_{3}\right), 3.10(\mathrm{q}, J=6.4 \mathrm{~Hz}, 2 \mathrm{H}$, $\left.\left(\left(\mathrm{CH}_{3}\right)_{2} \mathrm{NCH}_{2} \mathrm{CH}_{2} \mathrm{CH}_{2} \mathrm{NH}-\mathrm{C}=\mathrm{O}\right)\right), 2.67$ (d, $\left.J=5.1 \mathrm{~Hz}, 2 \mathrm{H}, \mathrm{CH}_{2} \mathrm{CONH}\right)$, 2.61 (t, $\left.2 \mathrm{H}, J=7.1 \mathrm{~Hz},\left(\mathrm{CH}_{3}\right)_{2} \mathrm{NCH}_{2} \mathrm{CH}_{2} \mathrm{CH}_{2} \mathrm{NH}-\mathrm{C}=\mathrm{O}\right), 2.56$ (s, $3 \mathrm{H}$, $\left.\left(\mathrm{CH}_{3}\right)_{2} \mathrm{~N}-\right), 2.26\left(\mathrm{~s}, 6 \mathrm{H}, 3-\mathrm{CH}_{3}, 6-\mathrm{CH}_{3}\right), 2.22\left(\mathrm{~s}, 6 \mathrm{H}, 2-\mathrm{CH}_{3}, 7-\mathrm{CH}_{3}\right), 1.79$ (p, J = 7.2 Hz, $\left.2 \mathrm{H},\left(\mathrm{CH}_{3}\right)_{2} \mathrm{NCH}_{2} \mathrm{CH}_{2} \mathrm{CH}_{2} \mathrm{NH}-\mathrm{C}=\mathrm{O}\right), 1.45$ (t, $6 \mathrm{H}, J=7.0 \mathrm{~Hz}$, $\mathrm{OCH}_{2} \mathrm{CH}_{3}$ ).

${ }^{13} \mathrm{C}$ NMR $\left(75 \mathrm{MHz}, \mathrm{CDCl}_{3}, 25^{\circ} \mathrm{C}, \mathrm{TMS}\right): \delta=172.4(\mathrm{C}(\mathrm{O}) \mathrm{NH}), 153.6$ (4a-C, 4b-C), 151.2 (1-Ar), 136.0 (3-Ar), 115.8 (2-Ar), 110.2 (9a-C), 107.3 (4-C), $63.8 \quad\left(\mathrm{OCH}_{2} \mathrm{CH}_{3}\right), \quad 55.5 \quad\left(\left(\mathrm{CH}_{3}\right)_{2} \mathrm{NCH}_{2} \mathrm{CH}_{2} \mathrm{CH}_{2} \mathrm{NH}-\mathrm{C}=\mathrm{O}\right), \quad 43.2$ $\left(\left(\mathrm{CH}_{3}\right)_{2} \mathrm{NCH}_{2} \mathrm{CH}_{2} \mathrm{CH}_{2} \mathrm{NH}-\mathrm{C}=\mathrm{O}\right), \quad 42.4 \quad\left(\mathrm{CH}_{2} \mathrm{CONH}\right), \quad 36.3$ $\left(\left(\mathrm{CH}_{3}\right)_{2} \mathrm{NCH}_{2} \mathrm{CH}_{2} \mathrm{CH}_{2} \mathrm{NH}-\mathrm{C}=\mathrm{O}\right), 26.9(9-\mathrm{C}), 25.3\left(\mathrm{CH}_{3}\right)_{2} \mathrm{NCH}_{2} \mathrm{CH}_{2} \mathrm{CH}_{2} \mathrm{NH}-$ $\mathrm{C}=\mathrm{O}), 20.3\left(3-\mathrm{CH}_{3}, 6-\mathrm{CH}_{3}\right), 11.4\left(2-\mathrm{CH}_{3}, 7-\mathrm{CH}_{3}\right)$.

HRMS (ESI): $m / z[M+H]^{+}$calcd. for $\mathrm{C}_{28} \mathrm{H}_{41} \mathrm{~N}_{2} \mathrm{O}_{4}$ : 469.30608; found 469.30440 .

\section{Acknowledgements}

The authors thank UNR (1BIO303 19/B450), CONICET (PIP GI 11220110100448) for financial support. PSF thanks CONICET for doctoral fellowship. GRL is member of the scientific staff of CONICET Argentina.

Keywords: PW2, amides, multicomponent reactions, iodine oxidative aromatization, 1,8-alkoxy-9H-Xanthen-9-yl-acetic acid alkyl esters.

[1] M. A. Biamonte, J. Wanner, K. G. Le Roch, Bioorganic Med. Chem. Lett. 2013, 23, 2829-2843.

[2] S. Edaye, S. J. Reiling, M. L. Leimanis, J. Wunderlich, $P$. Rohrbach, E. Georges, Mol. Biochem. Parasitol. 2014, 195 , 34-42.

[3] C. H. Sibley, Mol. Biochem. Parasitol. 2014, 195, 107-114. 
[4] C. Teixeira, N. Vale, B. Pérez, A. Gomes, J. Gomes, R. B, P. Gomes, Chem. Rev. 2014, 114, 11164-11220.

[5] Neena, S. Nain, V. Bhardwaj, R. Kumar, Pharm. Chem. J. 2015, 49, 254-258.

[6] K. R. M. Naidu, B. S. Krishna, M. A. Kumar, P. Arulselvan, S. I. Khalivulla, O. Lasekan, Molecules 2012, 17, 75437555.

[7] C. P. Wu, D. A. Van Schalkwyk, D. Taylor, P. J. Smith, K. Chibale, Int. J. Antimicrob. Agents 2005, 26, 170-175.

[8] K. Wiechmann, H. Müller, V. Huch, D. Hartmann, O. Werz, J. Jauch, Eur. J. Med. Chem. 2015, 101, 133-149.

[9] K. Chibale, M. Visser, V. Yardley, S. L. Croft, A. H. Fairlamb, Bioorganic Med. Chem. Lett. 2000, 10, 11471150.

[10] L. E. Luna, G. Seoane, R. M. Cravero, European J. Org. Chem. 2008, 1271-1277.

[11] L. E. Luna, R. M. Cravero, R. Faccio, H. Pardo, Á. W. Mombrú, G. Seoane, European J. Org. Chem. 2009, 30523057.

[12] R. M. Cravero, L. E. Luna, A. V. Barboza, Synthesis (Stuttg). 2011, 4027-4032.

[13] F. Zaragoza Dörwald, Organic Synthesis on Solid Phase, Wiley, 2002.

[14] V. Domingo, C. Prieto, L. Silva, J. M. L. Rodilla, J. F. Quílez Del Moral, A. F. Barrero, J. Nat. Prod. 2016, 79, 831-837.

[15] M. Jereb, D. Vražič, M. Zupan, Tetrahedron 2011, 67, 1355-1387.

[16] A. K. Banerjee, W. Vera, H. Mora, M. S. Laya, L. Bedoya, E. V. Cabrera, J. Sci. Ind. Res. (India). 2006, 65, 299-308.

[17] G. Majetich, S. Allen, Arkivoc 2010, 2010, 104-124.

[18] M. Lee, H. Kim, H. Rhee, J. Choo, ... Korean Chem. Soc. 2003, 24, 205-208.

[19] M. J. Mphahlele, T. B. Moekwa, Org. Biomol. Chem. 2005, 3, 2469-2475

[20] S. G. Hegde, A. M. Kassim, A. I. Kennedy, 2001, 57, 16891698.

[21] H. Togo, S. lida, Synlett 2006, 2159-2175.

[22] M. J. Mphahlele, Molecules 2009, 14, 4814-4837.

[23] V. R. Pattabiraman, J. W. Bode, Nature 2011, 480, 471-9.

[24] C. A. Horiuchi, Y. Nishio, D. Gong, T. Fujisaki, S. Kiji, Chem. Lett. 1991, 607-610.

[25] V. Sridharan, J. C. Mene, Synthesis (Stuttg). 2010, 38053849.

[26] J. W. Fisher, K. L. Trinkle, Tetrahedron Lett. 1994, 35 2505-2508.

[27] W. L. F. Armarego, C. L. L. Chai, Purification of laboratory chemicals, Butterworth Heinemann, 2009. 